\title{
The two-component giant radio halo in the galaxy cluster Abell 2142
}

\author{
T. Venturi ${ }^{1}$, M. Rossetti ${ }^{2}$, G. Brunetti ${ }^{1}$, D. Farnsworth ${ }^{3,4}$, F. Gastaldello ${ }^{2}$, S. Giacintucci ${ }^{5,6}$, D. V. Lal ${ }^{7}$, L. Rudnick ${ }^{3}$,
} T. W. Shimwell ${ }^{8}$, D. Eckert ${ }^{9}$, S. Molendi ${ }^{2}$, and M. Owers ${ }^{10,11}$

1 INAF-Istituto di Radioastronomia, via Gobetti 101, 40129 Bologna, Italy

e-mail: tventuri@ira.inaf.it

2 INAF-IASF-Milano, via Bassini 15, 20133 Milano, Italy

3 Minnesota Institute for Astrophysics, School of Physics and Astronomy, University of Minnesota, 116 Church Street SE, Minneapolis, MN, 55455, USA

4 Cray, Inc., 380 Jackson Street, Suite 210, St. Paul, MN 55101, USA

5 Naval Research Laboratory, Washington, DC 20375, USA

6 Department of Astronomy, University of Maryland, College Park, MD, 20742-2421, USA

7 National Centre for Radio Astrophysics, TIFR, Post Bag 3, Ganeshkhind, 411007 Pune, India

8 Leiden Observatory, Leiden University, PO Box 9513, 2300 RA Leiden, The Netherlands

9 Department of Astronomy, University of Geneva, ch. d'Écogia 16, 1290 Versoix, Switzerland

10 Australian Astronomical Observatory, PO Box 915, North Ryde, NSW 1670, Australia

11 Department of Physics and Astronomy, Maquarie University, NSW 2109, Australia

Received 4 November 2016 / Accepted 10 March 2017

\begin{abstract}
Aims. We report on a spectral study at radio frequencies of the giant radio halo in A $2142(z=0.0909)$, which we performed to explore its nature and origin. The optical and X-ray properties of the cluster suggest that A 2142 is not a major merger and the presence of a giant radio halo is somewhat surprising.

Methods. We performed deep radio observations of A 2142 with the Giant Metrewave Radio Telescope (GMRT) at $608 \mathrm{MHz}$, $322 \mathrm{MHz}$, and $234 \mathrm{MHz}$ and with the Very Large Array (VLA) in the 1-2 GHz band. We obtained high-quality images at all frequencies in a wide range of resolutions, from the galaxy scale, i.e. $\sim 5^{\prime \prime}$, up to $\sim 60^{\prime \prime}$ to image the diffuse cluster-scale emission. The radio halo is well detected at all frequencies and extends out to the most distant cold front in A 2142, about 1 Mpc away from the cluster centre. We studied the spectral index in two regions: the central part of the halo, where the X-ray emission peaks and the two brightest dominant galaxies are located; and a second region, known as the ridge (in the direction of the most distant south-eastern cold front), selected to follow the bright part of the halo and X-ray emission. We complemented our deep observations with a preliminary LOw Frequency ARray (LOFAR) image at $118 \mathrm{MHz}$ and with the re-analysis of archival VLA data at $1.4 \mathrm{GHz}$.

Results. The two components of the radio halo show different observational properties. The central brightest part has higher surface brightess and a spectrum whose steepness is similar to those of the known radio halos, i.e. $\alpha_{118 \mathrm{MHz}}^{1.78 \mathrm{GHz}}=1.33 \pm 0.08$. The ridge, which fades into the larger scale emission, is broader in size and has considerably lower surface brightess and a moderately steeper spectrum, i.e. $\alpha_{118 \mathrm{MHz}}^{1.78 \mathrm{GHz}} \sim 1.5$. We propose that the brightest part of the radio halo is powered by the central sloshing in A 2142, in a process similar to what has been suggested for mini-halos, or by secondary electrons generated by hadronic collisions in the ICM. On the other hand, the steeper ridge may probe particle re-acceleration by turbulence generated either by stirring the gas and magnetic fields on a larger scale or by less energetic mechanisms, such as continuous infall of galaxy groups or an off-axis (minor) merger.
\end{abstract}

Key words. galaxies: clusters: general - galaxies: clusters: individual: A 2142 - radio continuum: general

\section{Introduction}

Cluster mergers are the most energetic events in the Universe. With total energy outputs of order $10^{63}-10^{64} \mathrm{erg}$, mergers are a natural way to account for mass assembly: galaxy clusters form as a consequence of merger trees to reach and exceed masses of order $10^{15} M_{\odot}$. The gravitational energy released into the cluster volume during mergers deeply affects the dynamics of the galaxies and the properties of the thermal intracluster medium (ICM) and non-thermal relativistic particle and magnetic field emission (Brunetti \& Jones 2014). The impressive quality of the X-ray Chandra and XMM-Newton images (e.g. Markevitch et al. 2000; Rossetti et al. 2013) shows a variety of features in the hot ICM, such as cold fronts and shocks, which trace the cluster formation history and provide invaluable information concerning their dynamical state (see Markevitch \& Vikhlinin 2007). At the same time, the deep radio imaging achieved below $1 \mathrm{GHz}$ by the Giant Metrewave Radio Telescope (GMRT), and more recently by LOFAR, is shedding a new light on the properties of non-thermal components in galaxy clusters.

Radio halos represent the most spectacular non-thermal effects of cluster mergers. Radio halos are diffuse synchrotron radio sources that cover the whole cluster volume, i.e. up and beyond Mpc size. These halos have steep spectra, for which typical values are $\alpha \sim 1.2-1.3$, for $S \propto v^{-\alpha}$, where $S$ is the flux density and $\alpha$ is the spectral index of the synchrotron radio spectrum, and extremely low surface brightness of a fraction of $\mu \mathrm{Jy} \operatorname{arcsec}^{-2}$ (see the Feretti et al. 2012, for a recent observational review). 
Radio halos are not ubiquitous in galaxy clusters; only 30-40\% of massive clusters in the Universe $\left(M \geq 10^{15} M_{\odot}\right)$ host a radio halo. In those cases, the $1.4 \mathrm{GHz}$ radio power of the Mpc-scale halo correlates with both the cluster X-ray luminosity and mass (e.g. Liang et al. 2000; Brunetti et al. 2007; Basu 2012; Cassano et al. 2013; Cuciti et al. 2015). For those clusters without a radio halo, upper limits to the radio power derived from the GMRT radio halo survey are orders of magnitude below the correlation (Venturi et al. 2007; and 2008; Cassano et al. 2013; Kale et al. 2013 and 2015a).

The origin of giant radio halos is still a debated issue, however the connection between radio halos and cluster mergers is quantitatively supported by the distribution of clusters with and without radio halos as a function of a number of indicators of the X-ray substructure: giant radio halos are always found in unrelaxed clusters (Buote 2001; Cassano et al. 2010). On the other hand, a strong correlation is found between the cool-core strength in relaxed clusters and the presence of radio mini-halos; that is, diffuse cluster-scale sources that are smaller in size than giant radio halos (of the order of up to few hundreds of $\mathrm{kpc}$ ), which always encompass the radio emission from the cluster dominant galaxy, but whose origin is not closely related to the current cycle of activity (e.g. Bravi et al. 2016; Giacintucci et al. 2014; Kale et al. 2015b; Mittal et al. 2009).

A possible explanation for the origin of giant radio halos is the re-acceleration of in situ relativistic particles by turbulence injected into the ICM during cluster merger events (the so-called re-acceleration model; see Brunetti et al. 2001). Secondary (hadronic) models for the origin of the relativistic particles (e.g. Dennison 1980; Blasi \& Colafrancesco 1999) are currently less favoured because of the lack of detection of predicted $\gamma$-ray emission from galaxy clusters by the Fermi satellite, and because of the discovery of radio halos with ultra-steep spectra ( $\alpha \geq 1.5$ ), whose relativistic energy would exceed the thermal energy under the secondary model assumptions (see the prototype case of A 521; Brunetti et al. 2008). Mixed hadronic and re-acceleration models have been proposed and some level of radio emission is expected in "radio off-state clusters" (e.g. Brown et al. 2011; Brunetti \& Lazarian 2011; Zandanel et al. 2014).

Despite the statistical connection between radio halos and mergers, a few radio halos have been found in less disturbed systems. An example of these outliers is the giant radio halo in the strong cool-core cluster CL 1821+643 (Bonafede et al. 2014). A study of the X-ray morphological parameters of this cluster shows that it shares the same properties of galaxy clusters hosting a radio halo, suggesting that it may be a case of a cluster merger in which the cool core has been preserved (Kale $\&$ Parekh 2016). More recently, a giant radio halo has been reported in A 2261 and A 2390, neither of which is a major merger (Sommer et al. 2016). Current models predict that radio halos can also be generated in less disturbed systems, although with a probability that is significantly lower than in the case of massive major mergers (Cassano et al. 2006; and Brunetti \& Jones 2014, for a review). Such outliers are thus very important, as they may provide important constraints on the origin of radio halos, and probe a piece of the theoretical framework that is still poorly explored.

The galaxy cluster A $2142(z=0.0909)$ is another challenge to our understanding of the formation of giant radio halos.

A 2142 is massive $\left(M \sim 8.8 \times 10^{14} M_{\odot}\right.$, Cuciti et al. 2015) and is located at the centre of a supercluster (Einasto et al. 2015; Gramann et al. 2015) with ongoing accretion groups. It is the first object where cold fronts were discovered by Chandra (Markevitch et al. 2000). Recently, another cold front at the unprecedented distance of $\sim 1 \mathrm{Mpc}$ from the cluster centre was detected with XMM-Newton and studied by Rossetti et al. (2013), who proposed that large-scale sloshing was the responsible mechanism for its origin; this either resulted from the longterm evolution of central sloshing common in many relaxed clusters or from a merger of intermediate strength.

Diffuse radio emission on the scale of few hundred $\mathrm{kpc}$ around the two brightest cluster galaxies (BCGs) was detected by Giovannini \& Feretti (2000). A more recent study performed with the Green Bank Telescope (GBT) at $1410 \mathrm{MHz}$ shows that the size of this diffuse radio emission is $\sim 2 \mathrm{Mpc}$, extending even beyond the most distant cold front. The major axis of this giant radio halo is aligned in the same south-east direction where the large-scale cold front is located (Farnsworth et al. 2013). The GBT image shows that its surface brightness is very low, i.e. $\sim 0.2 \mu \mathrm{Jy} \operatorname{arcsec}^{-2}$, but the poor angular resolution does not allow a detailed comparison with the X-ray images. To overcome the resolution limitations of the GBT image and to study the spectral properties of this exceptional radio halo for comparison with the X-ray emission and optical information, we undertook a study with the GMRT at $608 \mathrm{MHz}, 322 \mathrm{MHz}$ and $234 \mathrm{MHz}$, and with the Karl Jansky Very Large Array (VLA) in the 1-2 GHz band. We complemented our analysis with LOw Frequency ARray (LOFAR) data at $118 \mathrm{MHz}$ and with archival VLA observations at $1.4 \mathrm{GHz}$.

In this paper we present the results of our work. The paper is organized as follows: in Sect. 2 we describe the observations and data analysis; the images are presented in Sect. 3; the spectral study is presented in Sect. 4; the results are discussed in Sect. 5; and the summary and future prospects are given in Sect. 6. In Appendix A we complement the information and report on the radio emission associated with the cluster galaxies. Throughout the paper we use the convention $S \propto v^{-\alpha}$. We assume a standard $\Lambda$ CDM cosmology with $H_{\mathrm{o}}=70 \mathrm{~km} \mathrm{~s}^{-1} \mathrm{Mpc}^{-1}$, $\Omega_{\mathrm{M}}=0.3, \Omega_{\mathrm{v}}=0.7$. At the cluster redshift $(z=0.0909)$ this corresponds to a scale of $1.705 \mathrm{kpc} /{ }^{\prime \prime}$ and to a luminosity distance $D_{\mathrm{L}}=418.6 \mathrm{Mpc}$.

\section{Observations and data reduction}

\subsection{Observations with the GMRT}

We observed A 2142 with the GMRT (Pune, India) in March 2013 at 608, 322, and $234 \mathrm{MHz}$. The logs of the observations are given in Table 1.

The data were collected in spectral-line mode at all frequencies, i.e. 256 channels at 322 and $608 \mathrm{MHz}$, and 128 channels at $234 \mathrm{MHz}$, with a spectral resolution of $125 \mathrm{kHz} /$ channel at $322 \mathrm{MHz}$ and $608 \mathrm{MHz}$, and $65 \mathrm{kHz} /$ channel at $234 \mathrm{MHz}$. The raw data were first processed with the software flagcal (Prasad \& Chengalur 2013; Chengalur 2013) to remove RFI and apply bandpass calibration, then further editing, self-calibration, and imaging were performed using the NRAO Astronomical Image Processing System (AIPS) package. The sources 3C 286 and 3C 48 were used as primary (amplitude) calibrators. In order to find a compromise between the size of the dataset and the need to minimize bandwidth smearing effects within the primary beam, after bandpass calibration the central channels in each individual dataset were averaged to 30, 39, and 26 channels of $\sim 1 \mathrm{MHz}$ each at $608 \mathrm{MHz}, 322 \mathrm{MHz}$, and $234 \mathrm{MHz}$, respectively.

At each frequency we performed multi-facet imaging, covering an area of $\sim 2.5^{\circ} \times 2.5^{\circ}$ at $234 \mathrm{MHz}, \sim 1.8^{\circ} \times 1.8^{\circ}$ at $322 \mathrm{MHz}$ and $\sim 1.4^{\circ} \times 1.5^{\circ}$ at $608 \mathrm{MHz}$, respectively. The field of A 2142 is extremely crowded, as is clear from Fig. 1, and includes many 
Table 1. Observations.

\begin{tabular}{clcccccc}
\hline \hline Array & Project ID & Obs. Date & $\begin{array}{c}v \\
\mathrm{MHz}\end{array}$ & $\begin{array}{c}\Delta v \\
\mathrm{MHz}\end{array}$ & $\begin{array}{c}\text { Time } \\
\mathrm{h}\end{array}$ & $\begin{array}{c}F W H M \\
\text { " } \times^{\prime \prime},{ }^{\circ}\end{array}$ & $\begin{array}{c}\mathrm{rms}^{a} \\
\mathrm{mJy} \mathrm{b}^{-1}\end{array}$ \\
\hline GMRT & $23 \_017$ & $23-03-13$ & 234 & 16 & 10 & $12.7 \times 11.0,68$ & 0.18 \\
& $23 \_017$ & $28-03-13$ & 322 & 32 & 10 & $9.8 \times 7.5,58$ & 0.13 \\
& 23_017 & $22-03-13$ & 608 & 32 & 10 & $5.4 \times 4.5,59$ & 0.03 \\
$\mathrm{VLA}^{b}$ & VLA11B-156 & $09-10-11$ & 1500 & 1000 & 1.5 & $c$ & $c$ \\
\hline
\end{tabular}

Notes. ${ }^{(a)}$ Value measured far from the field centre; ${ }^{(b)}$ the VLA observations consist of three pointings with the same set-up and exposure time (see Sect. 2.2). Here we give the total time over the three pointings; ${ }^{(c)}$ see Sect. 2.2. and Table 2.

strong sources, which had to be properly self-calibrated and cleaned to reach the targeted rms (see Table 1).

At each frequency we produced final images, over a wide range of resolutions and with different tapering and weighting schemes, to account for the complexity of the radio emission in the field. In particular, full resolution images were used to subtract the strongest radio sources at distances larger than $\sim 0.8^{\circ}-1.5^{\circ}$ (depending on the frequency) from the field centre, and then tapering and robust weighting were used to image the diffuse radio sources and the radio halo. Images with multiple resolutions were produced with resolutions ranging from $5.5^{\prime \prime} \times 4.5^{\prime \prime}$ to $39.1^{\prime \prime} \times 36.6^{\prime \prime}$ at $608 \mathrm{MHz}, 9.8^{\prime \prime} \times 7.5^{\prime \prime}$ to $53.4^{\prime \prime} \times 43.7^{\prime \prime}$ at $322 \mathrm{MHz}$, and $12.7^{\prime \prime} \times 11.0^{\prime \prime}$ to $45.19^{\prime \prime} \times 44.80^{\prime \prime}$ at $234 \mathrm{MHz}$.

Finally, to image the radio halo we first produced high resolution images using the $u-v$ spacings $>2 \mathrm{k} \lambda$. Then we used these to subtract the clean components of the discrete sources from all of the $u-v$ data, and imaged the residual emission with a taper and natural weighting (ROBUST $=+2$ in AIPS) to a resolution of $\sim 50^{\prime \prime}-60^{\prime \prime}$ at all frequencies. All images were primary beam corrected using the task PBCOR in AIPS. The shortest baselines in our datasets are $\sim 0.2 \mathrm{k} \lambda, \sim 0.1 \mathrm{k} \lambda$, and $\sim 0.07 \mathrm{k} \lambda$, respectively at $608 \mathrm{MHz}, 322 \mathrm{MHz}$, and $234 \mathrm{MHz}$. The largest detectable features are hence $17^{\prime}, 32^{\prime}$ and $44^{\prime}$, respectively.

The final rms values for the full resolution images are given in Table 1. At lower resolution we obtained rms $\sim 0.05 \mathrm{mJy} \mathrm{b}^{-1}$ at $608 \mathrm{MHz}\left(13.18^{\prime \prime} \times 11.06^{\prime \prime}\right), \sim 0.25 \mathrm{mJy} \mathrm{b}^{-1}$ at $322 \mathrm{MHz}$ $\left(14.80^{\prime \prime} \times 13.06^{\prime \prime}\right)$, and $\sim 0.3 \mathrm{mJy} \mathrm{b}^{-1}$ at $234 \mathrm{MHz}\left(19.46^{\prime \prime} \times\right.$ $\left.17.92^{\prime \prime}\right)$. We estimate that residual calibration errors at each frequency are within $4-5 \%$ at $608 \mathrm{MHz}$ and of the order of $10 \%$ at $234 \mathrm{MHz}$ and $322 \mathrm{MHz}$. We point out that the difference in the flux density between the Baars et al. (1977) scale adopted here and the Scaife \& Heald (2012) scale, suggested for observations at $v \leq 500 \mathrm{MHz}$, is of the order of few percent, hence within our final estimated errors. Figure 1 shows the full field of view at $234 \mathrm{MHz}$ and highlights the size of the fields imaged at $322 \mathrm{MHz}$ and $608 \mathrm{MHz}$.

\subsection{Jansky VLA observations}

A2142 was observed with the Karl G. Jansky VLA in D and $\mathrm{C}$ configurations at $1-2 \mathrm{GHz}$ as part of NRAO observing programme VLA11B-156. Three pointings were acquired to recover the full extent of the radio halo detected with the GBT (Farnsworth et al. 2013) with $28 \mathrm{~min}$ of integration time per pointing. Observations were made in spectral line mode with 16 spectral windows, each $64 \mathrm{MHz}$ wide, spread across the full $1-2 \mathrm{GHz}$ band. For technical reasons due to the recent VLA upgrade, only two seconds of every five second integration on source were recorded, which resulted in higher thermal
Table 2. Parameters of the full resolution VLA images.

\begin{tabular}{cccr}
\hline \hline $\begin{array}{c}v \\
\mathrm{MHz}\end{array}$ & $\begin{array}{c}\Delta \mathrm{MHz} \\
{ }^{\prime}\end{array}$ & $\begin{array}{c}F W M \\
\text { " } \times^{\prime \prime}\end{array}$ & $\begin{array}{r}\mathrm{rms} \\
\mathrm{mJy} \mathrm{b}^{-1}\end{array}$ \\
\hline 1380 & 250 & $40^{\prime \prime} \times 37^{\prime \prime}$ & 0.15 \\
1780 & 200 & $32^{\prime \prime} \times 29^{\prime \prime}$ & 0.07 \\
\hline
\end{tabular}

noise and less complete $u-v$ coverage. Standard data flagging and reduction techniques were performed with CASA, using the VLA calibrator sources J 1331+3030 (3C 286) and J 1609+2641 for flux and phase calibration, respectively. After editing for RFI, roughly $45 \%$ of the total bandwidth remained, yielding $\sim 450 \mathrm{MHz}$ over seven clean spectral windows. We created two images, one using $250 \mathrm{MHz}$ bandwidth around $1.38 \mathrm{GHz}$ and one using $200 \mathrm{MHz}$ around $1.78 \mathrm{GHz}$. The standard phase and amplitude calibration were successful enough that selfcalibration did not produce a significant improvement, so it was not used in the image presented here. Residual amplitude calibration errors are estimated to be within $3 \%$.

We used the multi-frequency multi-scale clean task in CASA (Rau \& Cornwell 2011) both to deconvolve and create a mosaic from the three pointings for each of the 1.38 and $1.78 \mathrm{GHz}$ maps. Correction for primary beam attenuation was performed using the CASA task impbcor.

To isolate the diffuse cluster emission we subtracted the contribution from radio galaxies, as follows. We used the $\mathrm{C}$ configuration $1.6 \mathrm{GHz}$ maps (resolution of $11^{\prime \prime}$, rms sensitivity of $\left.90 \mu \mathrm{Jy} \mathrm{b}^{-1}\right)$ to create masks of the location and extents of radio galaxies; these data were not calibrated accurately enough to directly subtract from the D configuration data. With these masks, we then performed an interactive single-scale clean of the $1.38 \mathrm{GHz}$ and $1.78 \mathrm{GHz}$ full resolution $\sim 40^{\prime \prime} \mathrm{D}$ configuration images until the radio galaxies were no longer visible. Model $u-v$ datasets representing the radio galaxy emission were created from these clean components and subtracted from the original D configuration $u-v$ data. The residual $u-v$ datasets were then imaged with multi-scale clean and corrected for the primary beam, convolving the final maps to $60^{\prime \prime}$ with an rms of $\sim 180 \mu \mathrm{Jy} \mathrm{b}^{-1}$ $\left(\sim 140 \mu \mathrm{Jy} \mathrm{b}^{-1}\right)$ at field centre for $1.38(1.78) \mathrm{GHz}$, to increase the signal-to-noise ratio of the diffuse emission. The largest detectable angular size of these observations is $\lesssim 1000^{\prime \prime}$ and $\lesssim 820^{\prime \prime}$, at $1.38 \mathrm{GHz}$ and $1.78 \mathrm{GHz}$, respectively.

\section{Radio emission from cluster galaxies}

The inner portion of the field of view of the GMRT observations, i.e. A 2142 itself, is shown in Figs. 2 and 3, where the contours of the $234 \mathrm{MHz}$ and $608 \mathrm{MHz}$ radio emission are overlaid on 


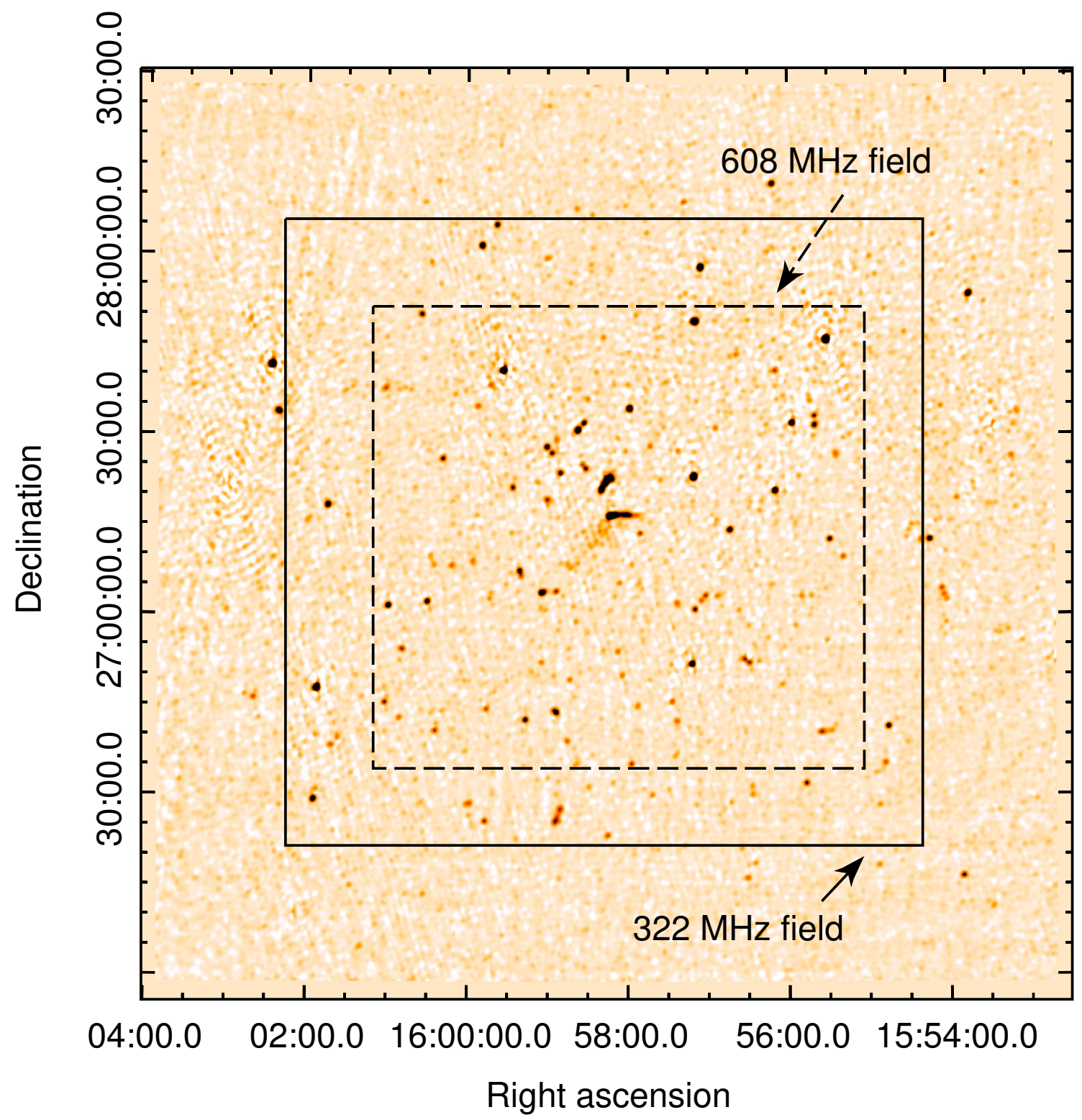

Fig. 1. Field of A 2142 at GMRT $234 \mathrm{MHz}$. The low resolution image is restored with a beam of $45.2^{\prime \prime} \times 40.8^{\prime \prime}$, PA $-37.9^{\circ}$. The continuous and dashed black boxes show the size of the $322 \mathrm{MHz}$ and $608 \mathrm{MHz}$ fields, respectively.

the red plate of the Digitized Sky Survey DSS-2 and the XMMNewton image, respectively.

The central region of A 2142 is dominated by the presence of two extended FRI radio galaxies (Fanaroff \& Riley 1974) with head-tail morphology (Sect. 3.1) and by diffuse emission coincident with the brightest part of the X-ray emission from the intracluster medium, which we classify as a giant radio halo (Sect. 4). Beyond these striking features, many radio sources in the field are associated with cluster galaxies.

Table A.1 reports the full sample of radio sources with optical counterpart at the redshift of A 2142 in the $608 \mathrm{MHz}$ field of view (Fig. 1). The list is based on the full resolution $608 \mathrm{MHz}$ image with a detection limit $S_{608 \mathrm{MHz}}=0.25 \mathrm{mJy}$ (i.e. $5 \sigma$ ) prior to the primary beam correction. For this reason, the radio source catalogue is not complete in radio power, whose detection limit increases away from the cluster centre. Flux density values at $234 \mathrm{MHz}$ have also been reported in the table. The radio galaxies presented in Sect. 3.1 are shown in Fig. A.1, where the full resolution $234 \mathrm{MHz}$ and $608 \mathrm{MHz}$ contours are overlaid on a
CFHT (Canadian French Hawaii Telescope) MegaCam $g$-band image.

\subsection{Radio galaxies at the cluster centre}

The most striking radio galaxies at the centre of A 2142 are two long tailed sources labelled T1 and T2 in Fig. 2. A zoom on each of them is shown in the upper left and upper right panels of Fig. A.1.

The source $\mathrm{T} 1$ is the radio galaxy B2 $1556+27$ (Colla et al. 1972, Owen et al. 1993), associated with a $m_{\mathrm{g}}=17.5$ cluster galaxy $(z=0.0955)$. A compact counterpart is visible in the XMM-Newton image. The head of this radio galaxy is coincident with the north-western cold front in the cluster. The length of the tail is $\sim 610 \mathrm{kpc}$. Figure A.1 clearly shows that the long and straight tail has small amplitude wiggles. The imaging process at all frequencies reveals that it is embedded in the diffuse emission of the radio halo. Its radio power is $\log P_{608 \mathrm{MHz}}=24.80 \mathrm{~W} \mathrm{~Hz}$, which is high for this class of 


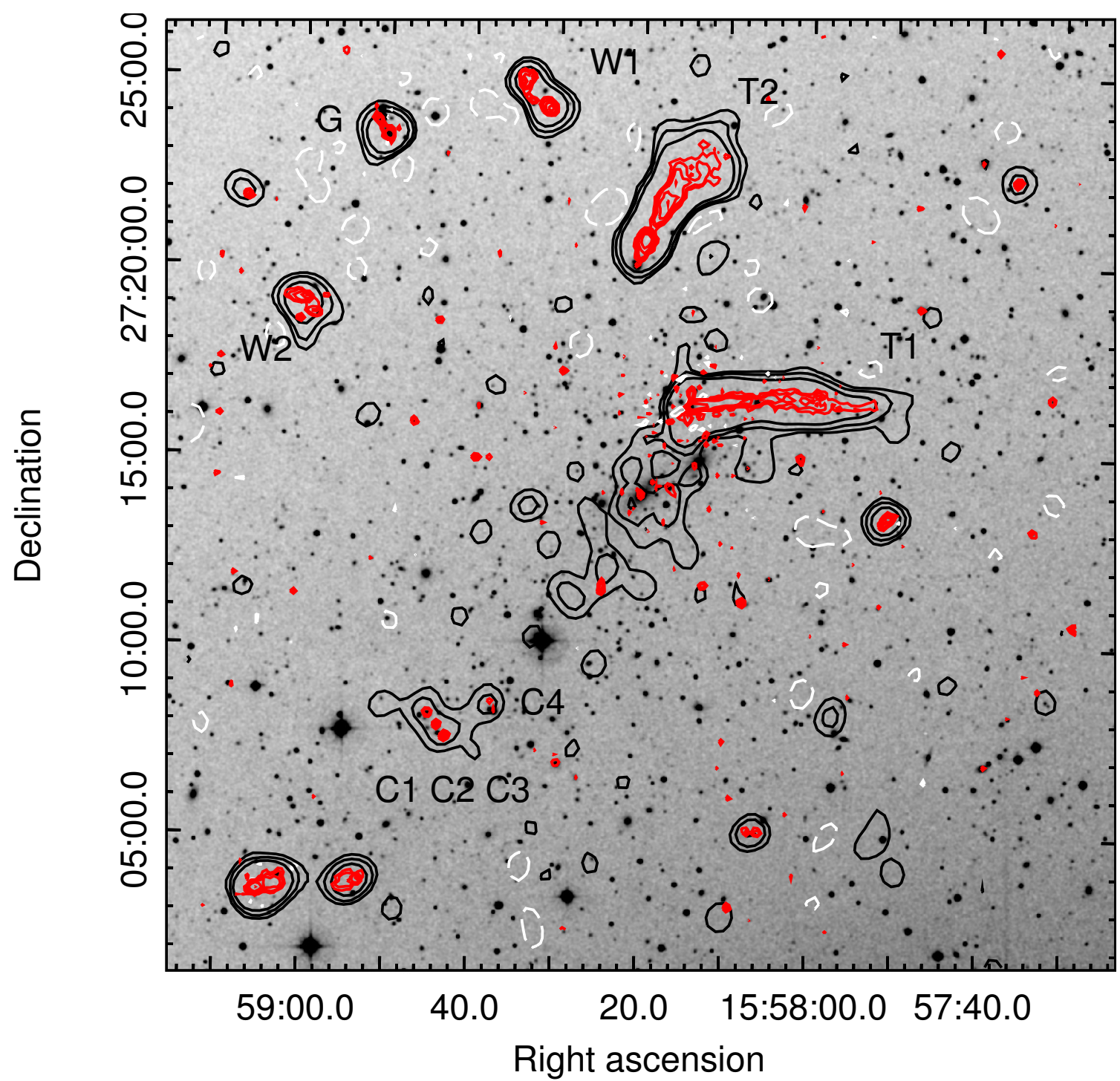

Fig. 2. Radio emission of A 2142 overlaid on the red plate of DSS-2. Black contours show a low resolution GMRT 234 MHz image restored with a beam of $45.2^{\prime \prime} \times 40.8^{\prime \prime}, \mathrm{PA}-37.9^{\circ}$ (same as Fig. 1); contour levels are $\pm 3,6,12 \mathrm{mJy} / \mathrm{b}$; and the rms in the image is $\sim 0.9 \mathrm{mJy} \mathrm{b}^{-1}$ far from the field centre (negative contours are white dashed). Red contours show the $608 \mathrm{MHz}$ full resolution image restored with a beam of 5.2" $\times 4.5^{\prime \prime}$, PA 52.6 contour levels are $\pm 0.1,0.2,0.4,0.8,1.6,6.4,25.6,102.4 \mathrm{mJy} \mathrm{b}^{-1}$; and the rms in the image is $\sim 35 \mu \mathrm{Jy} \mathrm{b}^{-1}$ far from the field centre. Negative contours are shown in white.

objects. We measure $\alpha_{234 \mathrm{MHz}}^{608 \mathrm{MHz}}=0.73 \pm 0.15$, which steepens to $\alpha_{608 \mathrm{MHz}}^{1400 \mathrm{MHz}}=1.04 \pm 0.11^{1}$.

The source $\mathrm{T} 2$ is associated with the galaxy 2MASX J $15582091+2720010\left(m_{\mathrm{g}}=16.6, z=0.0873\right.$ from the NASA/IPAC Extragalaxtic Database; NED), located north of the cluster centre and outside the brightest part of the X-ray emission, as is clear from Fig. 3. Its length is $\sim 370 \mathrm{kpc}$ and its radio power is $\log P_{608 \mathrm{MHz}}=24.51 \mathrm{~W} \mathrm{~Hz}^{-1}$. From Table A. 1 it is clear that T2 has a very steep integrated spectrum. If we complement our GMRT flux density measurements with the archival $1.4 \mathrm{GHz}$ data, we obtain $\alpha_{234 \mathrm{MHz}}^{608 \mathrm{MHz}}=1.21 \pm 0.14$, which steepens to $\alpha_{608 \mathrm{MHz}}^{1400 \mathrm{MHz}}=1.92 \pm 0.11$.

\footnotetext{
1 The VLA C+D configuration $1.4 \mathrm{GHz}$ observations are a re-analysis of project AG344. We produced images with angular resolution 24.1" $\times$ $21.9^{\prime \prime}$ and $\sim 38.6^{\prime \prime} \times 34.9^{\prime \prime}$ with rms of the order of $\sim 15 \mu \mathrm{Jy} \mathrm{b}^{-1}$ and $\sim 20 \mu \mathrm{Jy} \mathrm{b}^{-1}$, respectively. Individual source subtraction was performed following the same procedure described in Sect. 2.1 to obtain images of the diffuse emission.
}

Two wide-angle tail (WAT) sources are also present, and labelled W1 and W2 in Fig. 2. A zoom on each of them is shown in the left and right central panels of Fig. A.1, respectively. Interestingly, neither of these is located at the cluster redshift. The source $\mathrm{W} 1$ has a very faint optical counterpart $\left(m_{\mathrm{g}}=23\right.$ from NED) with $z_{\text {phot }}=0.574$; its flux density is $S_{608 \mathrm{MHz}}=37.99 \mathrm{mJy}$. The radio peak of W2 coincides with an X-ray source and has a very faint optical counterpart. Despite the presence of three very nearby cluster galaxies, an association with any of these seems unlikely. Considering that wide-angle tails are tracers of galaxy clusters (e.g. Giacintucci \& Venturi 2009; Mao et al. 2009) and that both W1 and W2 are associated with very faint objects, at least another cluster along the line of sight and behind A 2142 must be present.

The radio emission labelled $\mathrm{G}$ (bottom left panel of Fig. A.1) was presented in Eckert et al. (2014) at $608 \mathrm{MHz}$. It is a remarkable blend of discrete radio sources associated with a group at $z \sim 0.094$ located north-east of the cluster centre. The southern tip of the radio emission is coincident with the bright tip of the long $(\sim 800 \mathrm{kpc}) \mathrm{X}$-ray tail visible in Fig. 3, which has been 


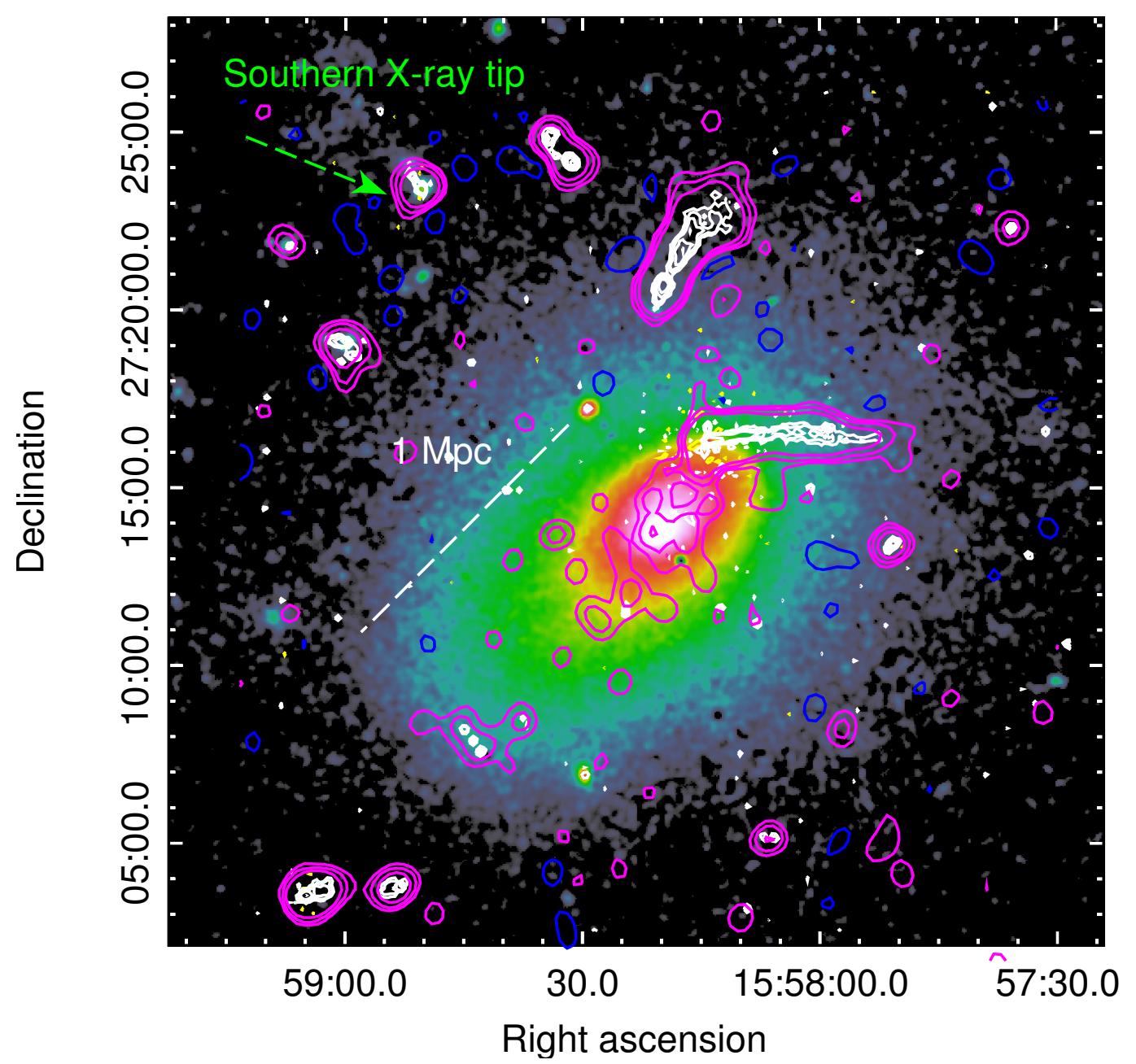

Fig. 3. Radio emission of A 2142 overlaid on the X-ray image from XMM-Newton. Magenta contours show a $234 \mathrm{MHz}$ low resolution image restored with a beam of $60^{\prime \prime} \times 60^{\prime \prime}$. Contours are drawn at $1.5,3,6,12,24,48 \mathrm{mJy} \mathrm{b}^{-1}$. Negatives contours are shown in blue $\left(-3 \mathrm{mJy} \mathrm{b}{ }^{-1}\right)$. The white contours show the $608 \mathrm{MHz}$ image (same contours and resolution as in Fig. 2).

interpreted as the signature of the infall of the group into A 2142 (Eckert et al. 2014 and 2017). We associate the southern peak of this emission with the brightest $\left(m_{\mathrm{g}}=16.1\right)$ galaxy in the group (Table A.1), even though the overlay shown in Fig. A.1 suggests that it could be a blend of emission from more galaxies.

The three compact radio galaxies labelled $\mathrm{C} 1, \mathrm{C} 2$, and $\mathrm{C} 3$ in Fig. 2 form another interesting group. They are aligned and associated with cluster galaxies of similar optical magnitude (in the range $m_{\mathrm{g}}=17.4-17.7$ from NED). A fourth cluster radio galaxy, $\mathrm{C} 4$, is located just west of this triplet, as seen in the bottom right panel of Fig. A.1.

The high resolution images at $608 \mathrm{MHz}$ reveal that some cluster galaxies embedded in the giant halo have associated radio emission. The most luminous BCG $\left(m_{\mathrm{g}}=16.2\right.$ and $\left.z=0.0904\right)$ hosts a faint radio source (see upper left panel of Fig. A.1). Moreover, a fainter cluster galaxy $\left(m_{\mathrm{g}}=18.8, z=0.0806\right)$ just north-west of the most luminous BCG and two more cluster galaxies located along the X-ray elongation from the BCGs to the $\mathrm{C} 1-\mathrm{C} 2-\mathrm{C} 3$ group show radio emission.

\subsection{Overall radio properties of the galaxies in A2142}

A total of 42 radio sources have an optical counterpart. These numbers refer to the region covered by the $608 \mathrm{MHz}$ observations. With exceptions made for T1 (GMRT J 155814+271619) and T2 (GMRT J 155820+272000), the two tailed radio galaxies at the cluster centre, all radio sources are either point-like or barely extended at the full resolution of the $608 \mathrm{MHz}$ image with radio powers in the range $21.85 \leq \log P_{608 \mathrm{MHz}} \lesssim 23 \mathrm{~W} \mathrm{~Hz}^{-1}$. These values suggest that the radio galaxy population of A 2142 may include both starburst galaxies and faint radio active nuclei. For the latter, the radio powers are well below the FRI/FRII division and are typical of the faint FR0 radio galaxies, whose radio morphology lacks extended emission in the form of radio lobes (Baldi et al. 2015, and references therein).

The radio emitting galaxies in A 2142 span a range of redshifts from $\sim 0.079$ to $\gtrsim 0.12$ (see Table A.1); this is consistent with the presence of multiple groups, as highlighted in the spectroscopic analysis performed by Owers et al. (2011) and in the structure analysis shown in Einasto et al. (2015). In Fig. A.2 we show the location of the cluster radio galaxies listed in Table A.1 with colour codes to highlight the different redshifts.

The wider field of view of the $234 \mathrm{MHz}$ (Fig. 1) includes many more radio sources associated with cluster galaxies. Starting from the visual inspection on DSS-2 and after consultation of the NED database we found 71 counterparts in the redishift range $z \sim 0.07-0.13$. These are mostly located south and east of the cluster centre, following the overall elongation of the cluster subgroups (Owers et al. 2011) and of the supercluster (Einasto et al. 2015). A full characterization of the population of radio 

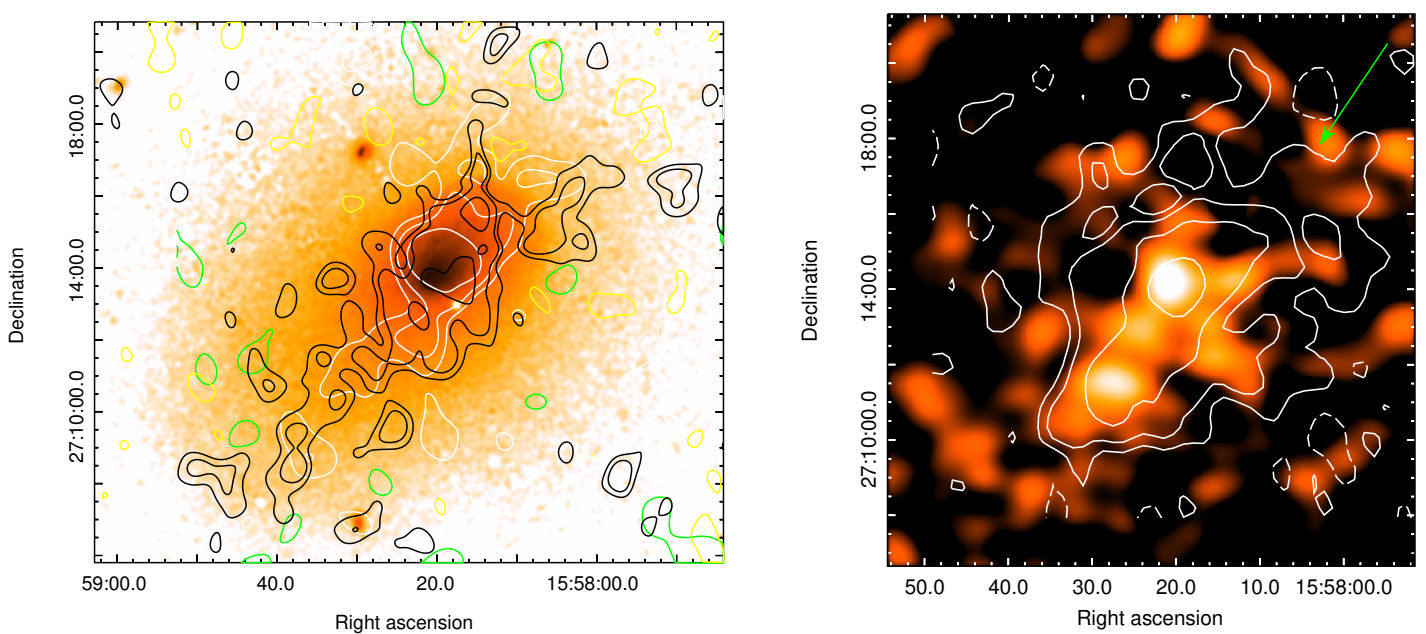

Fig. 4. Left panel: radio halo in A 2142 overlaid on the XMM-Newton emission. The $234 \mathrm{MHz}$ image is shown in black (negative contours in yellow). Contour levels are drawn at $\pm 2.5,4,8 \mathrm{mJy} \mathrm{b}^{-1}, \theta=44.86^{\prime \prime} \times 40.96^{\prime \prime}$, PA $-39^{\circ}$. The $608 \mathrm{MHz}$ emission is shown in white (negative contours shown in green). Contour levels are drawn at $\pm 0.6,1.2,2.4 \mathrm{mJy} \mathrm{b}^{-1}, \theta=50^{\prime \prime} \times 50^{\prime \prime}$. Both images were obtained after subtraction of the discrete sources from the $u-v$ data. Right panel: VLA contours at $1377 \mathrm{MHz}\left(\theta=60^{\prime \prime} \times 60^{\prime \prime}\right.$, contours are drawn at $\left.\pm 0.2,0.4,0.8,1.6 \mathrm{mJy} \mathrm{b}^{-1}\right)$ overlaid on the $322 \mathrm{MHz}$ GMRT image. Negative contours are drawn as dashed lines. The green arrow highlights the north-western extension (see Sect. 4.1)

sources in A 2142 is beyond the scope of this paper and will be presented in a future work.

\section{Radio halo}

\subsection{Morphology}

The diffuse extended emission in A 2142 is well visible in Figs. 1-3, and this emission is best highlighted in the low resolution images shown in the left and right panels of Fig. 4 (obtained after subtraction of the individual radio galaxies at each frequency; see Sects. 2.1 and 2.2), and in Fig. 5. Our images are suggestive of a multi-component cluster-scale emission, which all together we refer to as the radio halo. For our study we identify two regions, which are shown in the left panel of Fig. 6 and are named $\mathrm{H} 1$ and $\mathrm{H} 2$. The operational definition of these two regions is given in Sect. 4.2.

The region $\mathrm{H} 1$ is the brightest part of the halo, and this region is best highlighted in Fig. 5, which shows an intermediate resolution $608 \mathrm{MHz}$ image overlaid on the Chandra X-ray emission. The radio and X-ray peaks are coincident. This region was formerly classified as mini-halo (Giovannini \& Feretti 2000). Figure 5 clearly shows that it is confined by the innermost cold front, whose position is indicated by the green arrows. Even though the north-western boundary is more difficult to define, owing to the presence of the radio emission from T1, H1 does not seem to extend beyond the north-western cold front. This region has the same extent and boundaries at all frequencies.

The left panel of Fig. 4 shows the entire extended radio emission in A 2142 at $234 \mathrm{MHz}$ and $608 \mathrm{MHz}$ overlaid on the $\mathrm{X}$-ray emission detected by XMM-Newton, while the emission at $1.38 \mathrm{GHz}$ is given as contours in the right panel, overlaid with the $322 \mathrm{MHz}$ image. At all frequencies, the radio halo is elongated in the same north-west to south-east direction of the $\mathrm{X}$-ray emission from the intracluster gas, and covers the brightest X-ray ridge of emission out to the most distant cold front. Its largest angular size is $\sim 10^{\prime}$, i.e. $\sim 1 \mathrm{Mpc}$ at the cluster redshift. We define as $\mathrm{H} 2$ the ridge-like emission extending from $\mathrm{H} 1$ towards the most distant old front in the south-east direction. Inspection of both panels of Fig. 4 suggests that the surface

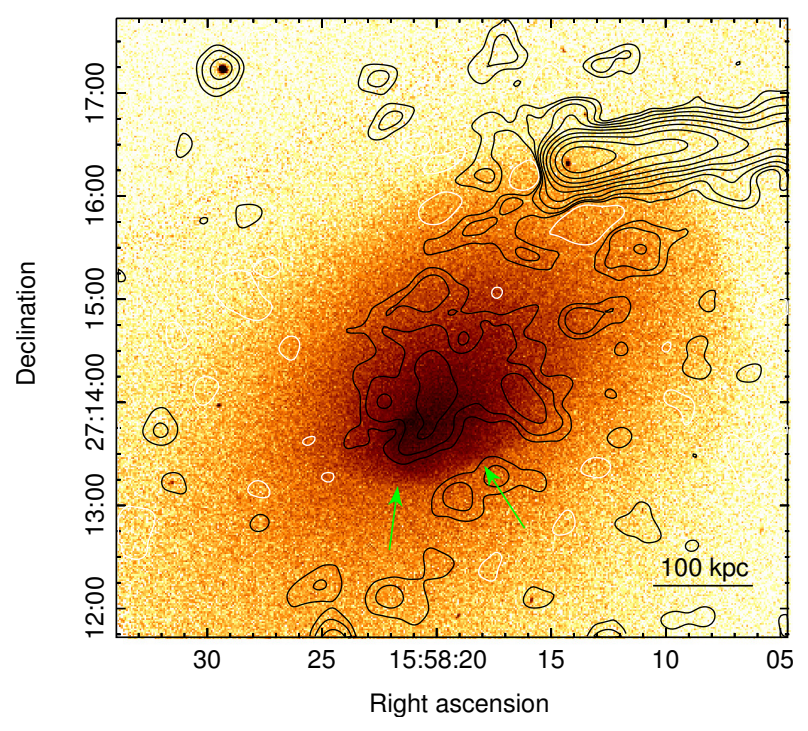

Fig. 5. Zoom on the central part of the radio halo. The GMRT $608 \mathrm{MHz}$ image (discrete radio sources not subtracted) at the resolution of $16.3^{\prime \prime} \times$ $14.2^{\prime \prime}, \mathrm{PA}-84.6^{\circ}$, is overlaid on Chandra. Contours are drawn at \pm 0.18 , $0.36,0.72,1.44,2.88 \mathrm{mJy} \mathrm{b}^{-1}$; positive contours are shown in black, negative contours white). The green arrows show the location of the inner SE cold front.

brightness distribution of this component is very different from $\mathrm{H} 1$. It is considerably more extended than $\mathrm{H} 1$ and lacks a central peak. The difference between $\mathrm{H} 1$ and $\mathrm{H} 2$ is confirmed by our analysis of the radio brightness profile across the two regions shown in the left panel of Fig. 6 (see Sect. 4.2 for details). The region $\mathrm{H} 1$ shows a regular profile with a prominent peak, following closely the brightness distribution of the hot gas. The profile of $\mathrm{H} 2$ is less regular, with a flat shoulder and a steeper profile far from the core, and shows no clear correlation with the X-ray profile. The north-western extension visible at $1.38 \mathrm{GHz}$ (right panel of Fig. 4) is not detected at lower frequencies and it is most likely due to incomplete subtraction of T1.

Finally, our VLA datasets detect further extended emission surrounding $\mathrm{H} 1$ and $\mathrm{H} 2$ (see the left panel of Fig. 6), which we 

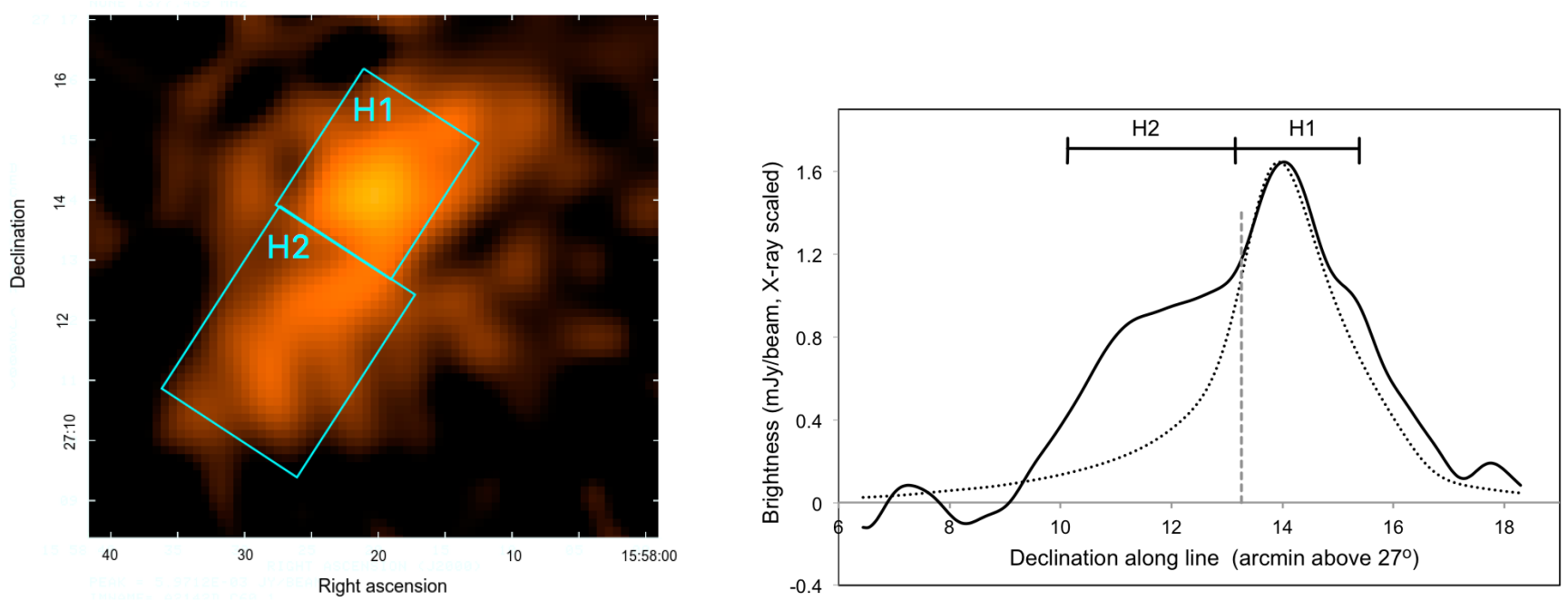

Fig. 6. Left panel: two regions $\mathrm{H} 1$ and $\mathrm{H} 2$ used for the evaluation of the integrated spectrum (see Sect. 4.2) shown on the 1.38 GHz VLA image at the resolution of $60^{\prime \prime}$. Right panel: slice of the surface brightness of the $1.38 \mathrm{GHz}$ image along the direction $-33^{\circ}$ (see Sect. 4.2), so that lengths along the slice can be calculated as (Dec2- Dec1)/cos(33d). The vertical grey dotted line shows the location of the south-east innermost cold front, while the XMM X-ray brightness profile convolved with the same resolution $\left(60^{\prime \prime}\right)$ is shown as a black dotted line.

interpret as the remains of the $2 \mathrm{Mpc}$ scale emission imaged with the GBT (Farnsworth et al. 2013), whose full extent is unrecovered in all our images. This clearly shows the limitations of interferometric observations, whose lack of zero spacings is a severe limit in imaging very low brightness extended and complex radio emission (as is the case of the radio halo in A 2142) at least out to $z \sim 0.1$.

In the next sections we refer to $\mathrm{H} 1$ as the central emission, $\mathrm{H} 2$ as the ridge, and the larger scale emission for the more diffuse emission detected with the VLA and GBT.

\subsection{Radio spectrum}

The complexity of the radio emission in A 2142 at all frequencies does not allow reliable imaging of the spectral index distribution throughout the radio halo. To overcome this difficulty and obtain some information about possible changes of the spectral properties, we derived the integrated spectrum of the radio halo in $\mathrm{H} 1$ and $\mathrm{H} 2$ (see the left panel of Fig. 6, where the two regions are overlaid on the VLA image).

To complement and further extend the frequency coverage of the GMRT and VLA observations we re-analysed $1.4 \mathrm{GHz}$ archival data in the $\mathrm{C}$ and $\mathrm{D}$ configuration (see Sect. 3.1) and used a preliminary image obtained with LOFAR at $118 \mathrm{MHz}$. The LOFAR HBA_DUAL_INNER 118-190 MHz data were recorded on April 192014 (project ID LC1_017). A subset of the target dataset in the band $118-124 \mathrm{MHz}$ was calibrated and imaged using the standard direction independent calibration procedure (see e.g. Shimwell et al. 2017). The resulting image, made from the visibilities from baselines shorter than $7 \mathrm{k} \lambda$, has a sensitivity of $3 \mathrm{mJy} \mathrm{b}^{-1}$, an angular resolution of $\sim 50^{\prime \prime}$ and the peak flux density measurements of compact sources are in agreement with the $150 \mathrm{MHz}$ TGSS ADR (Intema et al. 2016) survey to within $30 \%$. A full direction dependent calibration of the dataset (see e.g. van Weeren et al. 2016a,b; Williams et al. 2016; Hardcastle et al. 2016; and Shimwell et al. 2016) will be applied in the future to correct for ionospheric disturbances and improve the image fidelity, sensitivity and resolution, but this is beyond the scope of this paper.
Finally, we inspected the $74 \mathrm{MHz}$ image in the VLSS-Redux (VLA Low-Frequency Sky Survey) to check for the presence of diffuse radio emission at this frequency and found no clear emission above the noise level.

To determine the spectral indices for $\mathrm{H} 1$ and $\mathrm{H} 2$, we carried out the following steps: (a) we defined the $\mathrm{H} 1$ and $\mathrm{H} 2$ boundaries; (b) we adopted a procedure that was insensitive to variations in the detailed structures at different frequencies and that removed the varying amounts of flux from the $2 \mathrm{Mpc}$ components to measure the flux density of each component; and (c) we evaluated the $u-v$ coverage to ensure reliable flux estimates. We describe each of these below.

a) $\mathrm{H} 1$ and $\mathrm{H} 2$ definition - The extents of $\mathrm{H} 1$ and $\mathrm{H} 2$ were initially estimated by eye from greyscale images of the best images. To look at this more quantitatively, we calculated the average flux at each position along the major axis of the radio emission in a strip $160^{\prime \prime}$ wide along the minor axis. The slice of the surface brightness, obtained using the VLA 1.38 $\mathrm{GHz}$ image convolved to $60^{\prime \prime}$, is shown in the right panel of Fig. 6. Because $\mathrm{H} 1$ and $\mathrm{H} 2$ overlap, the division between them is somewhat arbitrary. It was first chosen as where H1 begins to significantly affect the shape of the $\mathrm{H} 2$ profile and then compared to the position of the cold front. The $\mathrm{H} 1, \mathrm{H} 2$ division is at the southern base of the cold front (see Fig. 5 and right panel of Fig. 6). The north-west boundary of H1 is approximately at its half-power level, as is the south-east boundary of $\mathrm{H} 2$. Because of the variations in the detailed structure at the different frequencies, the widths of the $\mathrm{H} 1$ and $\mathrm{H} 2$ regions were chosen to be the FWHM of the emission after smoothing by $160^{\prime \prime}$ along the major axis. For H1, we used the width of the bright core, rather than the lower brightness emission. The $\mathrm{H} 2$ profile is somewhat asymmetric, so the larger (SW) width was used. These procedures led to the length and width of the boxes as $160^{\prime \prime} \times 136^{\prime \prime}$ ( 6 square arcmin) for H1 and 216" $\times 160^{\prime \prime}$ (9.6 square arcmin) for H2, oriented at $-33 \mathrm{deg}$.

b) Flux density measurements - All images were first convolved to a resolution of $60^{\prime \prime}$, except for the VLSSr, which has a beam size of $75^{\prime \prime}$. The total flux densities in the H1 and $\mathrm{H} 2$ boxes were then measured as follows. For each 
T. Venturi et al.: The two-component giant radio halo in A 2142
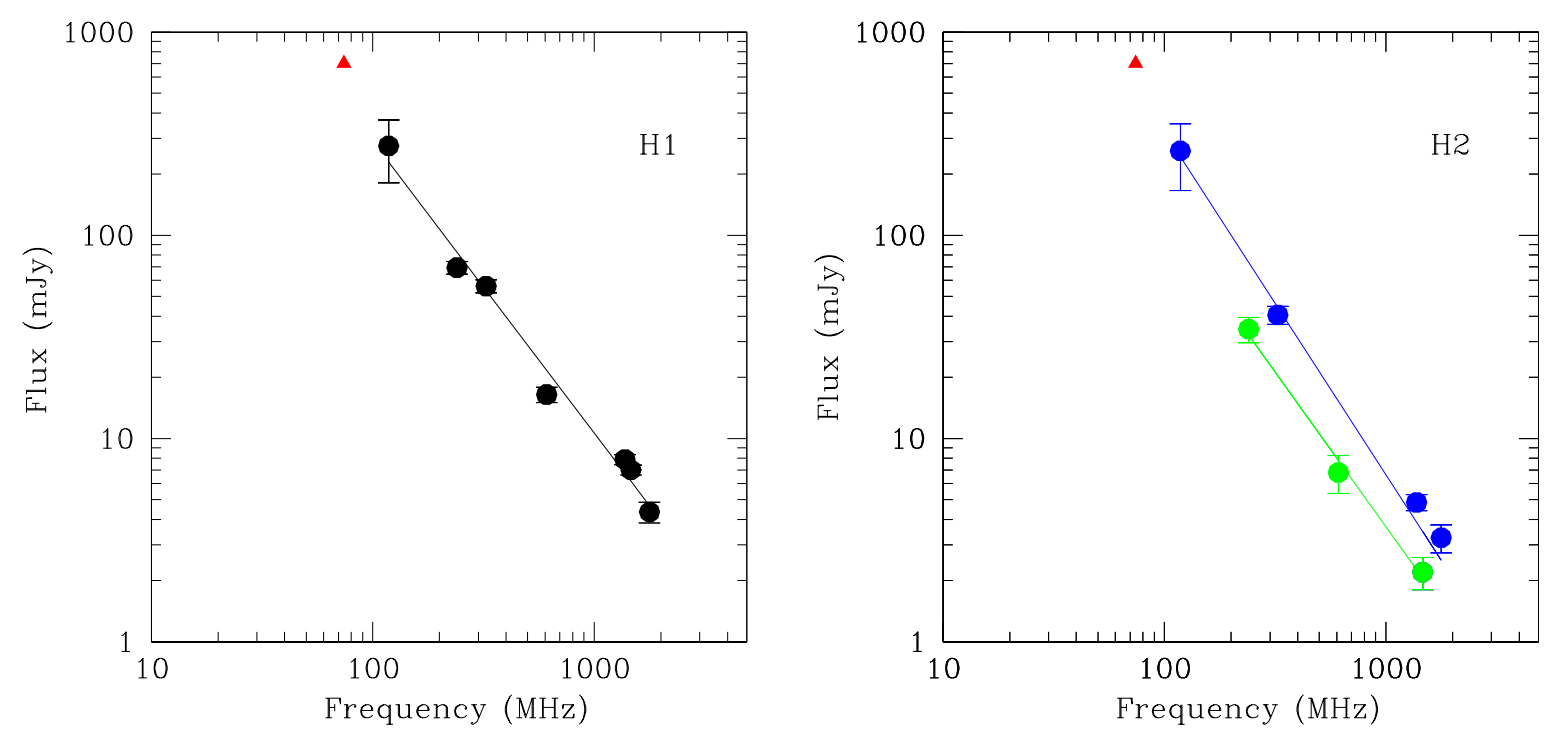

Fig. 7. Integrated spectrum of the radio halo. Left panel: plot of $\mathrm{H} 1$. The upper limit at $74 \mathrm{MHz}$ is shown as a red triangle. Right panel: plot of $\mathrm{H} 2$. Filled blue circles highlight the measurements with good $u-v$ coverage at short spacings; the remaining measurements are shown as filled green dots see Sect. 4.2). In both panels the weighted linear fits are shown with the same colour code and the upper limit at $74 \mathrm{MHz}$ is shown as a red triangle.

component, we calculated the running sum of the flux within a box fixed to the same length and width as $\mathrm{H} 1$ and $\mathrm{H} 2$, respectively. The box was slid along the line perpendicular to the major axis over a length of $1120^{\prime \prime}$ crossing the major axis (and $\mathrm{H} 1$ and $\mathrm{H} 2$, respectively) near the middle. We then measured both the off-source background level in each running sum, along with the peak flux above the background. These peak fluxes are reported in Table 3. The background level includes any small contributions from the much larger scale ( $20^{\prime}$ component detected in our single dish measurements), as well as any instrumental effects in the interferometer images. We then calculated the residual rms scatter in the offsource background of each running sum and report these as the errors for the peak fluxes. Such errors therefore reflect random noise and instrumental artifacts but do not reflect any systematic effects such as those due to $u-v$ coverage, which we discuss below. It is important to note that the H1 and $\mathrm{H} 2$ fluxes are not the "total flux" from each component, since they each have broad wings beyond their box widths. It is not clear whether these wings are extensions of $\mathrm{H} 1$ and $\mathrm{H} 2$ or part of the larger scale emission. However, since the boxes were kept the same at all frequencies, they provide a well-defined sample of the $\mathrm{H} 1$ and $\mathrm{H} 2$ emission. The right panel of Fig. 6 shows the surface brightness profile along the ridge oriented at $-33 \mathrm{deg}$. The clear difference in surface brightness and extent between $\mathrm{H} 1$ and $\mathrm{H} 2$ separately motivated our study of the total spectrum for these two regions.

c) Evaluation of the $u-v$ coverage - Despite the nominal largest detectable angular size of our observations at each frequency (see Sects. 2.1 and 2.2, for completeness note that the largest angular size detectable by LOFAR at $118 \mathrm{MHz}$ is $\sim 1^{\circ}$ ), because of their very low surface brightness none of our interferometer data sample well the largest scale $\left(20^{\prime}\right)$ emission seen with the single dish. We note that the total flux density detected with the VLA at $1377 \mathrm{MHz}$ is $23 \pm 2 \mathrm{mJy}$, which includes $\mathrm{H} 1, \mathrm{H} 2$, and the emission beyond these two regions until they fade into the background, while the $1410 \mathrm{MHz}$ flux density measured in the GBT image is $\sim 55 \mathrm{mJy}$ (see Farnsworth et al. 2013). However, the partial sampling of this very diffuse component, which varies from frequency to frequency, can lead to elevated background levels around $\mathrm{H} 1$ and $\mathrm{H} 2$. The determination of a background level in the 1D cuts, as discussed above, removes any such effects. The total spectrum of $\mathrm{H} 1$ and $\mathrm{H} 2$ between $118 \mathrm{MHz}$ and $1.778 \mathrm{GHz}$ is shown in the left and right panel of Fig. 7, respectively. We point out that $\mathrm{H} 2$, whose largest extent along its major axis $\left(216^{\prime \prime}\right)$ is well within the nominal largest detectable structure, is not sufficiently well sampled in our interferometric images owing to to its surface brightness, which is considerably lower than H1. In particular, the inner portion of the $u-v$ coverage (within $1 \mathrm{k} \lambda$ ) is much better sampled at $118 \mathrm{MHz}$ (LOFAR), $322 \mathrm{MHz}$ (GMRT), $1.38 \mathrm{GH}$, and $1.78 \mathrm{GHz}$ (VLA-D). For this reason we refer to those images as to the well-sampled (we are using this or good coverage) images. By contrast, the $u-v$ coverage of the $1.4 \mathrm{GHz}$ archival VLA and the $608 \mathrm{MHz}$ and $234 \mathrm{MHz}$ GMRT data is poorer on the shortest spacings and some of the flux density from $\mathrm{H} 2$ is missing. This problem is well known and its effect has been quantified in earlier works on giant radio halos (see Brunetti et al. 2008; and Venturi et al. 2008). The flux density measurements for $\mathrm{H} 2$ are plotted separately in the right panel of Fig. 7.

A weighted mean square fit provides $\alpha_{118 \mathrm{MHz}}^{1.78 \mathrm{GHz}}=1.33 \pm 0.08$ for $\mathrm{H} 1$ and $\alpha_{118 \mathrm{GHz}}^{1.78 \mathrm{MHz}}=1.42 \pm 0.08$ for $\mathrm{H} 2$. The difference is only marginal. However, it becomes more significant if we take the different $u-v$ coverage into account. A fit of the two sets of measurements for $\mathrm{H} 2$ separately (filled green and filled blue dots in the right panel of Fig. 7), provides $\alpha_{118 \mathrm{MHz}}^{1.78 \mathrm{GHz}}=1.55 \pm 0.08$. The flux density upper limits at $74 \mathrm{MHz}$ are consistent with the spectral index both for $\mathrm{H} 1$ and $\mathrm{H} 2$. The future analysis of the LOFAR data will allow us to obtain better constraints.

\section{Origin of the radio halo}

The observations presented in this work confirm that in many respects A 2142 is a case study of galaxy clusters. Our most important results is the finding that the radio halo consists of two regions with different morphological and spectral properties. 
Table 3. Radio halo flux density.

\begin{tabular}{lrr}
\hline \hline Region & $\begin{array}{r}\text { Frequency } \\
\text { MHz }\end{array}$ & $\begin{array}{r}\text { Flux density } \\
\text { mJy }\end{array}$ \\
\hline $\mathrm{H} 1$ & 1778 & $4.4 \pm 0.5$ \\
& 1465 & $7.0 \pm 0.5$ \\
& 1377 & $7.9 \pm 0.4$ \\
& 608 & $16.5 \pm 1.4$ \\
& 322 & $56.2 \pm 4.1$ \\
& 234 & $69.3 \pm 4.9$ \\
& 118 & $275.0 \pm 94.1$ \\
& 74 & $<700$ \\
\hline $\mathrm{H} 2$ & 1778 & $3.3 \pm 0.5$ \\
& 1465 & $2.2 \pm 0.4$ \\
& 1377 & $4.9 \pm 0.4$ \\
& 608 & $6.8 \pm 1.4$ \\
& 322 & $40.5 \pm 4.1$ \\
& 234 & $34.5 \pm 4.9$ \\
& 118 & $260.0 \pm 94.1$ \\
74 & $<700$ \\
\hline
\end{tabular}

Our analysis, which spreads over a frequency range of more than one order of magnitude $(118-1.78 \mathrm{GHz})$, suggests that the region of emission extending south-east of the cluster core $(\mathrm{H} 2$; see left panel of Fig. 6) has a lower surface brightness, is broader in size, and has a moderately steeper spectrum than the brighter, more compact region in the core (H1). In this section we discuss the possible origin of such differences in the framework of the cluster dynamics, as inferred from its broadband properties. In Fig. 8 we show all the observational information that is relevant to the discussion.

\subsection{The central emission}

The radio emission observed in the core of A 2142 (H1) is bounded by the two inner cold fronts that are detected on the 100-200 kpc scale, as clear from Figs. 5 and 8. This may suggest that this emission traces the dissipation of the energy produced by the sloshing of the low-entropy gas oscillating between the two inner cold fronts. This scenario is similar to what has been suggested for the origin of mini-halos in cool-core clusters (e.g. Mazzotta \& Giacintucci 2008; ZuHone et al. 2013). Two BCGs are present in A2142 (see Figs. 8 and A.1) and the sloshing in the core may be due to the perturbation induced by a gasless minor merger with the group associated with the secondary BCG (Owers et al. 2011). These two BCGs may also play a role in the origin of the radio emission. At present only the most luminous BCG hosts a radio source (Sect. 3.1, Table A.1 and Fig. A.1), but it is likely that over the last Gyr or so both BCGs have released relativistic particles and magnetic fields in the core region. During this timescale relativistic electrons could be advected by turbulent motions in the sloshing gas. The very weak correlation between the radio power of the BCG and that of the mini-halo found for a sizeable sample of mini-halo clusters (Govoni et al. 2009; Giacintucci et al. 2014) is indeed suggestive of the fact that the central AGN activity may not be powering the radio emission directly, but it is the most likely source of seed electrons for reacceleration in the ICM.

In order to cover a distance of the order of $100 \mathrm{kpc}$ in $1 \mathrm{Gyr}$, the spatial diffusion coefficient due to turbulent transport should be of the order of $D \sim \delta V_{L} L \sim 2 \times 10^{30} \mathrm{~cm}^{2} \mathrm{~s}^{-1}$ (Brunetti $\&$ Jones 2014, and references therein), implying a velocity of the turbulent eddies of $\sim 100 \mathrm{~km} \mathrm{~s}^{-1}$ on a scale of about $50 \mathrm{kpc}$. These requirements are indeed consistent with those measured in simulations of gas sloshing and those necessary for turbulent reacceleration to maintain (or to re-accelerate) radio emitting electrons in these regions (ZuHone et al. 2013); in addition, values of the same magnitude range have been derived for the Perseus cluster (Hitomi collaboration 2016).

\subsection{The ridge}

The right panel of Fig. 6 clearly shows that the surface brightness and extent of the radio emission in the ridge (H2) are very different from those in the core region (H1). The clear separation between these two components suggests that they might originate from different mechanisms or that they trace different evolutionary stages of the same phenomenon. On the scale of the ridge the cluster appears unrelaxed with a clear elongation in the south-eastern direction. The radio emission follows the spatial distribution of the X-ray emitting gas and extends up to the cold front located at $1 \mathrm{Mpc}$ south-east of the core. On these scales the cluster may have been perturbed by a number of processes that induce gas sloshing and generate the external cold front. The detection of a bridge of low-entropy gas between the central region of the cluster and the most distant cold front is in support of this picture (Rossetti et al. 2013). It is likely that the gas and magnetic fields in this region have been moderately perturbed and stirred. We can speculate that this induces turbulence that cascades on smaller scales and damps into particle re-acceleration and fast magetic reconnection, which are two interconnected processes if incompressible turbulence is considered (Brunetti \& Lazarian 2016).

The low luminosity of the ridge and of the 2 Mpc scale emission, and the moderately steeper spectrum of the ridge, suggest that the energy budget that becomes available to the non-thermal components in $\mathrm{H} 2$ is smaller than that of classical giant radio halos, that indeed are generated during major merger events. An alternative possibility is that the radio emission is very old and marks the switched off phase of the radio halo, when merger turbulence is dissipated at later times. Both cases are possible for massive clusters that show intermediate properties between merging and relaxed systems (e.g. Cassano et al. 2006; Brunetti et al. 2009; Donnert et al. 2013). This is indeed the case of A 2142. As a matter of fact, this radio halo is considerably underluminous compared to the correlation between radio power and cluster mass (Cassano et al. 2013; Cuciti et al. 2017), even considering the GBT measurement in Farnsworth et al. (2013, see Sects. 4.2 and 6).

Different scenarios can explain the origin of the perturbations induced in the ICM. A possibility is that the three cold fronts detected in X-rays (see their location in Fig. 8) are generated by a single event (Rossetti et al. 2013). In this case, H1 and H2 may probe the evolution of this phenomenon on different scales and/or times and may trace different levels of perturbations and magnetic field strength that are present in the ICM. The symmetry of the three cold fronts and the way they encompass $\mathrm{H} 1$ and $\mathrm{H} 2$ is in support of this scenario.

Another possibility is that the ridge traces a turbulent region that is generated by the continuous accretion of subhalos along the $\mathrm{S}-\mathrm{E}$ direction. This hypothesis may be supported by the fact that optical data show the presence of several groups of galaxies that trace a large-scale filament in the S-E direction (Owers et al. 2011; Einasto et al. 2015), and group accretion in A 2142 is caught in action (Eckert et al. 2014). 


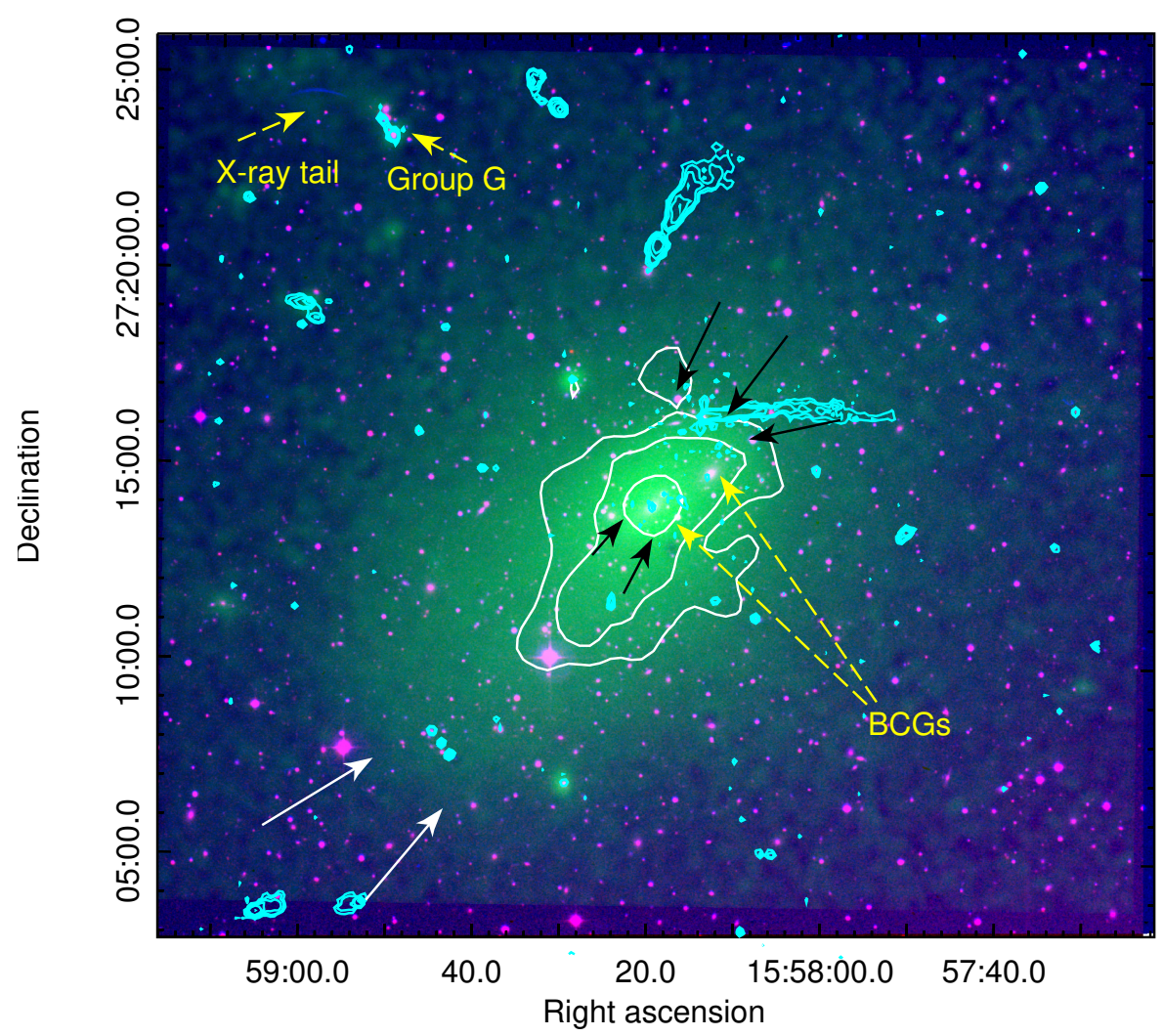

Fig. 8. Multi-band image of A 2142. The optical information from the combined red and blue plates of DSS-2 is shown in purple, the X-ray emission from XMM-Newton is shown in green, radio contours at $608 \mathrm{MHz}$ are shown in cyan (same image and contour levels as in Figs. 2 and 3 ), the emission from the radio halo at $1.38 \mathrm{GHz}$ is shown as white contours (same image as in the right panel of Fig. 6, contour levels drawn at $0.4,0.8,1.6 \mathrm{mJy} / \mathrm{b}$ ). The three cold fronts are indicated by the black and white arrows. The innermost cold front is coincident with the highest radio contour of the radio halo and the northernmost cold front is coincident with the lowest radio contours. For completeness, the location of the BCGs, of Group G and the X-ray tail discussed in Eckert et al. (2014) are also shown.

Relativistic hadrons and their secondaries generated via inelastic collisions in the ICM may also contribute to the origin of $\mathrm{H} 1$ and $\mathrm{H} 2$. Following the scenario proposed for radio halos by Zandanel et al. (2014), the halo in A 2142 may experience a transition from a hadronic emission component, dominating the emission in the core region (H1), to a mainly leptonic (re-acceleration) component, responsible for $\mathrm{H} 2$ and the large-scale emission. This scenario would qualitatively match the differences in the observed properties of $\mathrm{H} 1$ and $\mathrm{H} 2$. Inefficient re-acceleration in the ridge, as explained above, would produce a low-luminosity signal with steeper spectrum, whereas secondaries generated in the dense core would explain the higher brightness of $\mathrm{H} 1$ and its flatter spectrum.

It is interesting to note that the two-component radio halo in A 2142 is not unique. A similar situation has been found for the diffuse emission in A 2319 (Storm et al. 2015).

\section{Summary and conclusions}

Our radio observations of A 2142 show that the cluster is extremely interesting at radio wavelengths.

We confirm that the giant radio halo in the cluster extends $\sim 1 \mathrm{Mpc}$ in the NW-SE direction out to the region where the most distant cold front is located. It is one of the few cases of a giant radio halo in a cluster that is not a major merger (Bonafede et al. 2014; Sommer et al. 2016), thus it has the potential to provide important constraints on the origin of these sources.

The overall properties show that the radio halo consists of two different components. The first component is a bright and fairly compact central region $(\mathrm{H} 1)$ that is coincident with the brightest part of the X-ray, which is confined by the two inner cold fronts and covers the volume where the two BCGs are also located; the second component is a broader emission with lower surface brightness, which we named the ridge (H2) and extends out to the most distant cold front. The very large-scale emission imaged by the GBT at $1410 \mathrm{MHz}$ (Farnsworth et al. 2013 ) is undetected in all our interferometric images. The spectral properties of these two components, derived from $118 \mathrm{MHz}$ to $1.78 \mathrm{GHz}$, show some differences. The region $\mathrm{H} 1$ has a steep spectrum with $\alpha_{234 \mathrm{MHz}}^{1.478 \mathrm{GHz}}=1.33 \pm 0.08$, while the spectrum of $\mathrm{H} 2$ is moderately steeper, i.e. $\alpha_{118 \mathrm{MHz}}^{1.78 \mathrm{GHz}} \sim 1.5$, if we account for the relevance of the $u-v$ coverage of our set of observations in recovering the extent and flux density of this component.

The radio halo in A 2142 is significantly underluminous if compared to classical radio halos hosted in clusters with similar mass: it is almost a factor of 20 below the correlation in Cassano et al. (2013) if we consider the $1.4 \mathrm{GHz}$ radio power of $\mathrm{H} 1$ and $\mathrm{H} 2$ presented in this work. Even using the GBT measurement, which should be considered an upper limit, we obtain $P_{1.4 \mathrm{GHz}}=1.15 \times 10^{24} \mathrm{~W} \mathrm{~Hz}^{-1}$, i.e. a factor of $\sim 4-5$ lower than the correlation at the cluster mass, $M \sim 8.8 \times 10^{14} M_{\odot}$ (see Fig. 7 in Bernardi et al. 2016, for an updated version of the plot).

We suggest that two different mechanisms could be at the origin of the complex diffuse radio emission in A 2142. We propose that on the core scale $(\mathrm{H} 1)$ the emission is powered by mechanisms that are similar to those considered for radio minihalos. On larger scales (H2), however, the emission is powered by mechanisms that are similar to those considered for classical 
giant radio halos, but less powerful given that the dynamical properties of A 2142 are indeed less extreme than those of major merging systems. In this case turbulence may be generated by stirring of gas and magnetic fields on the large scale or by off-axis minor mergers. Alternatively, the properties of the radio halo may be interpreted as due to the transition between a hadronic (secondary) component in the core and a leptonic (turbulent re-acceleration) component on the larger scale of the ridge.

The phenomenology observed both in the X-rays and in the radio suggests that $A 2142$ is a suitable target to understand, at the same time, the origin and evolution of cold fronts on different scales and the connection between radio halos and mini-halos.

Our work confirms that the study of the origin of clusterscale radio sources is crucial to improve our understanding of the complex phenomena at play during the processes of cluster mergers and group accretion in the Universe.

Acknowledgements. T.V. and G.B. acknowledge partial support from PRININAF 2014. L.R. and D.F. acknowledge partial support from the US National Science Foundation under grant AST-1211595 to the University of Minnesota. TS acknowledges support from the ERC Advanced Investigator programme NewClusters 321271 . Basic research in radio astronomy at the Naval Research Laboratory is supported by 6.1 base funding. We thank the staff of the GMRT who have made these observations possible. GMRT is run by the National Centre for Radio Astrophysics of the Tata Institute of Fundamental Research. The National Radio Astronomy Observatory is a facility of the National Science Foundation operated under cooperative agreement by Associated Universities, Inc. The Very Large Array is operated by the National Radio Astronomy Observatory, which is a facility of the National Science Foundation operated under cooperative agreement by Associated Universities, Inc. This research has made use of the NASA/IPAC Extragalactic Database (NED) which is operated by the Jet Propulsion Laboratory, California Institute of Technology, under contract with the National Aeronautics and Space Administration. LOFAR, the Low Frequency Array designed and constructed by ASTRON, has facilities in several countries, which are owned by various parties (each with their own funding sources), and that are collectively operated by the International LOFAR Telescope (ILT) foundation under a joint scientific policy. This research used the facilities of the Canadian Astronomy Data Centre operated by the Nationa Research Council of Canada with the support of the Canadian Space Agency.

\section{References}

Baars, J. W. M., Genzel, R., Pauliny-Toth, I. I. K., et al. 1977, A\&A, 61, 99 Baldi, R. D., Capetti, A., \& Giovannini, G. 2015, A\&A, 576, A38 Basu, K. 2012, MNRAS, 421, L112 Bernardi, G., Venturi, T., Cassano, R., et al., 2016, MNRAS, 456, 1259 Blasi, P., \& Colafrancesco, S. 1999, Astropart. Phys., 12, 169 Bonafede, A., Intema, H., Brüggen, M., et al. 2014, MNRAS, 444, L44 Bravi, L., Gitti, M., \& Brunetti, G. 2016, MNRAS, 455, L41

Brown, S., Emerick, A., Rudnick, L., et al. 2011, ApJ, 740, L28 Brunetti, G., \& Lazarian, A. 2011, MNRAS, 410, 127 Brunetti, G., \& Jones, T. J. 2014, Inter. J. Mod. Phys. D., 23, 4
Brunetti, G., \& Lazarian, A. 2016, MNRAS, 458, 2584 Brunetti, G., Setti, G., Feretti, L., et al. 2001, MNRAS, 320, 365 Brunetti, G., Venturi, T., Dallacasa, D., et al. 2007, ApJ, 670, L5 Brunetti, G., Giacintucci, S., Cassano, R., et al. 2008, Nature, 455, 944 Brunetti, G., Cassano, R., Dolag, K., et al. 2009, A\&A, 507, 661 Buote, D. A. 2001, ApJ, 553, L15

Cassano, R., Brunetti, G., \& Setti, G. 2006, MNRAS, 327, 557

Cassano, R., Ettori, S., Giacintucci, S., et al. 2010, ApJ, 721, L82 Cassano, R., Ettori, S., Brunetti, G., et al. 2013, ApJ, 777, 141 Chengalur, J. N. 2013, NCRA Technical Report NCRA/COM/001 (April 2013) Colla, G., Fanti, C., Fanti R., et al. 1972, A\&AS, 7, 1

Cuciti, V., Cassano, R., Brunetti, G., et al. 2015, A\&A, 580, A97

Cuciti, V., Brunetti, G., van Weeren, R., et al. 2017, A\&A, submitted Dennison, B. 1980, ApJ, 293, L93

Donnert, J., Dolag, K., Brunetti, G., et al. 2013, MNRAS, 429, 3564

Eckert, D., Molendi, S., Owers, M., et al. 2014, A\&A, 570, A119

Eckert, D., Gaspari, M., Owers, M. S., et al. 2017, A\&A, in press DOI: $10.1051 / 0004-6361 / 201730555$

Einasto, M., Gramann, M., Saar, E., et al. 2015, A\&A, 580, A69

Fanaroff, B. L., \& Riley, J. M. 1974, MNRAS, 167, 31

Farnsworth, D., Rudnick, L., Brown, S., et al. 2013, ApJ, 779, 189

Feretti, L., Giovannini, G., Govoni, F., et al. 2012, A\&ARv, 20, 54

Giacintucci, S., \& Venturi, T. 2009, A\&A, 505, 55

Giacintucci, S., Markevitch, M., Venturi, T., et al. 2014, ApJ, 781, 9

Giovannini, G., \& Feretti, L. 2000, New Astron., 5, 335

Govoni, F., Murgia, M., Markevitch, M., et al. 2009, A\&A, 499, 371

Gramann, M., Einasto, M., Heinämäki, P., et al. 2015, A\&A, 581, 135

Hardcastle, M. J., Gürkan, G., van Weeren, R. J., et al. 2016, MNRAS, 462, 1910 Hitomi Collaboration 2016, Nature, 535, 117

Intema, H. T., Jagannathan, P., Mooley, K. P., et al. 2017, A\&A, 598, A78

Liang, H., Hunstead, R. W., Birkinshaw, M., et al. 2000, ApJ, 544, 686

Kale, R., \& Parekh, V. 2016, MNRAS, 459, 2940

Kale, R., Venturi, T., Giacintucci, S., et al. 2013, A\&A, 557, A99

Kale, R., Venturi, T., Giacintucci, S., et al. 2015a, A\&A, 579, A92

Kale, R., Venturi, T., Cassano, R., et al. 2015b, A\&A, 581, A23

Mao, M.Y., Johnston-Hollitt, M., Stevens, J. B., et al. 2009, MNRAS, 392, 1070 Markevitch, M., \& Vikhlinin, A. 2007, Phys. Rep., 443, 1

Markevitch, M., Ponman, T. J., Nulsen, P. E. J., et al. 2000, ApJ, 541, 542

Mazzotta, P., \& Giacintucci, S. 2008, ApJ, 675, L9

Mittal, R., Hudson, D. S., Reipreich, T. H., et al. 2009, A\&A, 501, 835

Owen, F. N., White, \& R. A., Ge, J. 1993, ApJS, 87, 135

Owers, M. S., Nulsen, P. E., \& Couch, W. J. 2011, ApJ, 741, 122

Prasad, J., \& Chengalur, J. N. 2012, Exp. Astron., 33, 157

Rau, U., \& Cornwell, T. J. 2011, A\&A, 532, A71

Rossetti, M., Eckert, D., De Grandi, S., et al. 2013, A\&A, 556, A44

Scaife, A. M. M., \& Heald, G. H. 2012, MNRAS, 423, L30

Shimwell, T. W., Luckin, J., Brüggen, M., et al. 2016, MNRAS, 459, 277

Shimwell, T. W., Röttgering, H. J. A., Best, P. N., et al. 2017, A\&A, 598, A104

Sommer, M. W., Basu, K., Intema, H., et al. 2017, MNRAS, 466, 996

Storm, E., Jeltema, T. E., \& Rudnick, L. 2015, MNRAS, 448, 2495

van Weeren, R. J., Williams, W. L., Hardcastle, M. J., et al. 2016a, ApJS, 223, 2

van Weeren, R. J., Brunetti, G., Brüggen, M., et al. 2016b, ApJ, 818, 204

Venturi, T., Giacintucci, S., Brunetti, G., et al. 2007, A\&A, 463, 937

Venturi, T., Giacintucci, S., Dallacasa, D., et al. 2008, A\&A, 484, 327

Williams, W. L., van Weeren, R. J., Röttgering, H. J. A., et al. 2016, MNRAS, 460, 2385

Zandanel, F., Pfrommer, C., \& Prada, F. 2014, MNRAS, 438, 124

ZuHone, J., Markevitch, M., Brunetti, G., et al. 2013, ApJ, 762, 78 
T. Venturi et al.: The two-component giant radio halo in A 2142

\section{Appendix A: Optical identifications}

In this Appendix we report the full list of optical counterparts belonging to A 2142 in the same field shown in Fig. 2, and radio/optical overlays both for the extended radio galaxies and for those groups where multiple radio emission has been detected.

Table A.1. Optical identifications with cluster galaxies.

\begin{tabular}{|c|c|c|c|c|c|c|}
\hline \#, GMRT name & $\alpha_{\mathrm{J} 2000}$ radio & $\delta_{\mathrm{J} 2000}$ radio & $S_{608 \mathrm{MHz}}(\mathrm{mJy})$ & $S_{234 \mathrm{MHz}}(\mathrm{mJy})$ & $\log P_{608 \mathrm{MHz}}(\mathrm{W} / \mathrm{Hz})$ & Notes \\
\hline Optical Catalogue & $\alpha_{\mathrm{J} 2000} \mathrm{opt}$ & $\delta_{\mathrm{J} 2000} \mathrm{opt}$ & $m_{\mathrm{g}}$ & \multirow{3}{*}{6.52} & $z$ & \multirow{3}{*}{$\mathrm{P}$} \\
\hline \#1, GMRT-J 155636+270041 & 155636.99 & 270041.7 & 1.67 & & 22.55 & \\
\hline WISEPC & 155637.07 & 270039.7 & 15.9 & & 0.091117 & \\
\hline \#2, GMRT-J $155642+273324$ & 155642.94 & 273324.1 & 3.18 & \multirow[t]{2}{*}{5.71} & 22.80 & \multirow[t]{2}{*}{$\mathrm{P}$} \\
\hline 2MASX & 155642.96 & 273324.6 & 17.0 & & 0.088777 & \\
\hline \#3, GMRT-J 155646+270015 & 155646.20 & 270015.2 & 0.53 & \multirow[t]{2}{*}{-} & 21.99 & \multirow[t]{2}{*}{$\mathrm{P}$} \\
\hline SDSS & 155646.25 & 270015.4 & 18.9 & & 0.085227 & \\
\hline \#4, GMRT-J 155700+273102 & 155700.56 & 273102.2 & 1.87 & \multirow[t]{2}{*}{4.42} & 22.50 & \multirow[t]{2}{*}{$\mathrm{P}$} \\
\hline 2MASX & 155700.56 & 273102.7 & 17.8 & & 0.082248 & \\
\hline \#5, GMRT-J 155703+271812 & 155703.29 & 271812.8 & 1.53 & \multirow[t]{2}{*}{3.65} & 22.54 & \multirow[t]{2}{*}{$\mathrm{P}$} \\
\hline 2MASX & 155703.34 & 271812.7 & 16.8 & & 0.094222 & \\
\hline \#6, GMRT-J $155708+273519$ & 155708.97 & 273519.0 & 2.92 & \multirow[t]{2}{*}{6.58} & 22.79 & \multirow[t]{2}{*}{$\mathrm{P}$} \\
\hline SDSS & 155708.92 & 273519.3 & 17.6 & & 0.123953 & \\
\hline \#7, GMRT-J 155709+2702434 & 155709.30 & 270243.0 & 0.75 & \multirow[t]{2}{*}{1.88} & 22.31 & \multirow[t]{2}{*}{$\mathrm{P}$} \\
\hline SDSS & 155709.43 & 270246.6 & 18.6 & & 0.102941 & \\
\hline \#8, GMRT-J 155714+272608 & 155714.12 & 271608.6 & 1.68 & \multirow[t]{2}{*}{3.29} & 22.60 & \multirow[t]{2}{*}{$\mathrm{P}$} \\
\hline SDSS & 155714.14 & 271607.9 & 18.4 & & 0.095882 & \\
\hline \#9, GMRT-J 155722+270111 & 155722.11 & 270111.8 & 0.57 & \multirow[t]{2}{*}{2.74} & 22.19 & \multirow[t]{2}{*}{$\mathrm{P}$} \\
\hline SDSS & 155722.00 & 270113.0 & 18.3 & & 0.103162 & \\
\hline \#10, GMRT-J 155739+270655 & 155739.93 & 270655.7 & 0.60 & \multirow[t]{2}{*}{-} & 22.14 & \multirow[t]{2}{*}{$\mathrm{P}$} \\
\hline 2MASX & 155739.24 & 270656.7 & 17.5 & & 0.095269 & \\
\hline \#11, GMRT-J 155739+272249 & 155739.84 & 272249.7 & 0.34 & \multirow[t]{2}{*}{-} & 21.86 & \multirow[t]{2}{*}{$\mathrm{P}$} \\
\hline 2MASX & 155739.91 & 272248.9 & 17.4 & & 0.091306 & \\
\hline \#12, GMRT-J 155744+270121 & 155744.90 & 270121.3 & 0.52 & \multirow[t]{2}{*}{-} & 22.10 & \multirow[t]{2}{*}{$\mathrm{P}$} \\
\hline SDSS & 155744.61 & 270119.0 & 22.2 & & 0.097427 & \\
\hline \#13, GMRT-J 155745+274042 & 155745.95 & 274042.3 & 1.59 & \multirow[t]{2}{*}{5.36} & 22.51 & \multirow[t]{2}{*}{$\mathrm{P}$} \\
\hline WISPEC & 155745.93 & 274043.6 & 17.3 & & 0.089887 & \\
\hline \#14, GMRT-J 155747+271857 & 155747.07 & 271857.3 & 0.88 & \multirow[t]{2}{*}{1.71} & 22.36 & \multirow[t]{2}{*}{$\mathrm{P}$} \\
\hline 2MASX & 155746.93 & 271856.6 & 17.4 & & 0.100987 & \\
\hline \#15, GMRT-J 155801+271500 & 155801.15 & 271500.4 & 1.10 & \multirow[t]{2}{*}{2.05} & 22.31 & $\mathrm{P}$ \\
\hline 2MASX & 155801.28 & 271500.8 & 17.8 & & 0.085613 & \\
\hline \#16, GMRT-J 155807+271112 & 155807.90 & 271112.5 & 1.75 & 3.44 & 22.68 & $\mathrm{P}$ \\
\hline SDSS & 155807.93 & 271112.1 & 17.8 & & 0.102417 & \\
\hline
\end{tabular}

Notes. Errors on the radio flux density are of the order of $4-5 \%$ at $608 \mathrm{MHz}$, and of the order of $10 \%$ at $234 \mathrm{MHz}$. The optical information has been taken from the NASA Extragalactic Database (NED). Notes are as follows: $\mathrm{P}=$ point-like source, NAT = narrow-angle tail, WAT $=$ wide-angle tail. ${ }^{(\star)}$ Position of optical identification; $\left.{ }^{(}\right)$Position of peak flux density. The sequence number for each source is used in Fig. A.2 to show the position on the optical plate. 
Table A.1. continued.

\begin{tabular}{|c|c|c|c|c|c|c|}
\hline \#, GMRT name & $\alpha_{\mathrm{J} 2000}$ radio & $\delta_{\mathrm{J} 2000}$ radio & $S_{608 \mathrm{MHz}}(\mathrm{mJy})$ & $S_{234 \mathrm{MHz}}(\mathrm{mJy})$ & $\log P_{608 \mathrm{MHz}}(\mathrm{W} / \mathrm{Hz})$ & Notes \\
\hline Optical Catalogue & $\alpha_{\mathrm{J} 2000} \mathrm{opt}$ & $\delta_{\mathrm{J} 2000} \mathrm{opt}$ & $m_{\mathrm{g}}$ & \multirow{3}{*}{-} & $z$ & \multirow{3}{*}{$\mathrm{P}$} \\
\hline \#17, GMRT-J 155812+271536 & 155812.39 & 271536.5 & 0.34 & & 21.85 & \\
\hline SDSS & 155812.23 & 271537.3 & 19.9 & & 0.090876 & \\
\hline \#18, GMRT-J 155814+271619 & 155814.31 & 271619.0 & 272.71 & \multirow[t]{2}{*}{550.11} & 24.80 & \multirow{2}{*}{$\mathrm{NAT}^{\star}-\mathrm{T} 1$} \\
\hline SDSS & 155814.31 & 271619.0 & 17.5 & & 0.095546 & \\
\hline \#19, GMRT-J 155816+271412 & 155816.56 & 271412.5 & 0.83 & \multirow[t]{2}{*}{1.91} & 22.22 & \multirow[t]{2}{*}{$\mathrm{P}$} \\
\hline SDSS & 155816.58 & 271411.5 & 18.1 & & 0.089338 & \\
\hline \#20, GMRT-J 155818+271421 & 155818.69 & 271421.5 & 0.47 & \multirow[t]{2}{*}{-} & 21.88 & \multirow[t]{2}{*}{$\mathrm{P}$} \\
\hline 2MASX & 155818.75 & 271421.0 & 17.8 & & 0.080353 & \\
\hline \#21, GMRT-J 155819+271400 & 155819.93 & 271400.5 & 1.00 & \multirow[t]{2}{*}{ - } & 22.32 & \multirow[t]{2}{*}{$\mathrm{P}$} \\
\hline 2MASX & 155820.02 & 271400.0 & 16.2 & & 0.090369 & \\
\hline \#22, GMRT-J 155820+272000 & 155820.84 & 272000.7 & 158.64 & \multirow[t]{2}{*}{501.47} & 24.51 & \multirow[t]{2}{*}{$\mathrm{Tail}^{\star}-\mathrm{T} 2$} \\
\hline 2MASX & 155820.91 & 272001.0 & 16.6 & & 0.089553 & \\
\hline \#23, GMRT-J 155824+271127 & 155824.20 & 271127.5 & 2.60 & \multirow[t]{2}{*}{5.05} & 22.74 & \multirow[t]{2}{*}{ Ext. $^{\star}$} \\
\hline SDSS & 155824.50 & 271125.5 & 18.0 & & 0.091530 & \\
\hline \#24, GMRT-J 155829+271713 & 155829.38 & 271713.9 & 0.90 & \multirow[t]{2}{*}{1.46} & 22.27 & \multirow[t]{2}{*}{$\mathrm{P}$} \\
\hline SDSS & 155829.36 & 271714.2 & 17.1 & & 0.089875 & \\
\hline \#25, GMRT-J 155829+270654 & 155829.70 & 270654.4 & 0.92 & \multirow[t]{2}{*}{0.96} & 22.22 & \multirow[t]{2}{*}{$\mathrm{P}$} \\
\hline 2MASX & 155829.55 & 270655.0 & 17.8 & & 0.084849 & \\
\hline \#26, GMRT-J 155831++265633 & 155831.70 & 265633.4 & 0.77 & \multirow[t]{2}{*}{1.99} & 22.15 & \multirow[t]{2}{*}{$\mathrm{P}$} \\
\hline 2MASX & 155831.73 & 265632.1 & 17.3 & & 0.085579 & \\
\hline \#27, GMRT-J 155837+270818 & 155837.01 & 270818.4 & 0.53 & \multirow[t]{2}{*}{2.01} & 22.04 & \multirow[t]{2}{*}{$\mathrm{P}$} \\
\hline 2MASX & 155837.04 & 270817.1 & 17.3 & & 0.090295 & \\
\hline \#28, GMRT-J, 155839+271618 & 155839.17 & 271618.4 & 0.38 & \multirow[t]{2}{*}{-} & 21.88 & \multirow[t]{2}{*}{$\mathrm{P}$} \\
\hline 2MASX & 155839.29 & 271618.1 & 17.6 & & 0.089194 & \\
\hline \#29, GMRT-J 155842+270736 & 155842.85 & 270736.3 & 3.73 & \multirow[t]{2}{*}{8.86} & 22.85 & \multirow[t]{2}{*}{$\mathrm{P}$} \\
\hline WISEPC & 155842.84 & 270736.5 & 17.4 & & 0.086434 & \\
\hline \#30, GMRT-J 155843+272254 & 155843.59 & 272254.3 & 0.39 & - & 21.90 & $\mathrm{P}$ \\
\hline SDSS & 155843.57 & 272253.2 & 18.7 & & 0.089284 & \\
\hline \#31, GMRT-J 155843+270752 & 155843.75 & 270752.8 & 2.23 & 4.30 & 22.63 & $\mathrm{P}$ \\
\hline SDSS & 155843.70 & 270752.8 & - & & 0.085881 & \\
\hline \#32, GMRT-J 155844+270812 & 155844.87 & 270812.3 & 1.65 & 3.42 & 22.58 & $\mathrm{P}$ \\
\hline WISEPC & 155844.97 & 270812.4 & 17.7 & & 0.094569 & \\
\hline \#33, GMRT-J 155844+271831 & 155844.13 & 271831.8 & 0.89 & 2.49 & 22.15 & $\mathrm{P}$ \\
\hline SDSS & 155844.15 & 271831.7 & 18.3 & & 0.079943 & \\
\hline \#34, GMRT-J 155845+263851 & 155845.42 & 263851.3 & 25.16 & 24.30 & 23.81 & $\mathrm{P}$ \\
\hline 2MASX & 155845.46 & 263851.0 & 16.3 & & 0.1000 & \\
\hline \#35, GMRT-J 155846+271552 & 155846.93 & 271552.8 & 1.20 & 2.95 & 22.30 & $\mathrm{P}$ \\
\hline SDSS & 155846.96 & 271552.8 & 18.0 & & 0.081561 & \\
\hline
\end{tabular}


T. Venturi et al.: The two-component giant radio halo in A 2142

Table A.1. continued.

\begin{tabular}{|c|c|c|c|c|c|c|}
\hline \#, GMRT name & $\alpha_{\mathrm{J} 2000}$ radio & $\delta_{\mathrm{J} 2000}$ radio & $S_{608 \mathrm{MHz}}(\mathrm{mJy})$ & $S_{234 \mathrm{MHz}}(\mathrm{mJy})$ & $\log P_{608 \mathrm{MHz}}(\mathrm{W} / \mathrm{Hz})$ & Notes \\
\hline Optical Catalogue & $\alpha_{\mathrm{J} 2000} \mathrm{opt}$ & $\delta_{\mathrm{J} 2000} \mathrm{opt}$ & $m_{\mathrm{g}}$ & \multirow{3}{*}{4.16} & $z$ & \\
\hline \#36, GMRT-J 155849+273639 & 155849.69 & 272639.2 & 2.06 & & 22.57 & $\mathrm{P}$ \\
\hline WISEPC & 155849.65 & 272640.3 & 17.0 & & 0.084872 & \\
\hline \#37, GMRT-J 155850+273324 & 155850.46 & 272324.2 & 31.89 & \multirow[t]{2}{*}{53.89} & 23.85 & \multirow[t]{2}{*}{$\mathrm{Ext}^{\diamond}-\mathrm{G}$} \\
\hline WISEPC & 155850.39 & 272324.5 & 16.1 & & 0.093601 & \\
\hline \#38, GMRT-J 155851+272349 & 155851.92 & 272349.7 & 3.25 & \multirow[t]{2}{*}{6.37} & 22.91 & \multirow[t]{2}{*}{$\mathrm{P}$} \\
\hline 2MASX & 155851.89 & 272350.3 & 17.2 & & 0.098645 & \\
\hline \#39, GMRT-J 155855+272124 & 155855.85 & 272124.1 & 0.41 & \multirow[t]{2}{*}{-} & 21.98 & \multirow[t]{2}{*}{$\mathrm{P}$} \\
\hline & 155855.7 & 272123 & 18.9 & & 0.0952906 & \\
\hline \#40, GMRT-J 155903+272140 & 155903.85 & 272140.5 & 0.33 & \multirow[t]{2}{*}{0.98} & 21.83 & \multirow[t]{2}{*}{$\mathrm{P}$} \\
\hline SDSS & 155903.74 & 272137.7 & 19.2 & & 0.089533 & \\
\hline \#41, GMRT-J 155920+271138 & 155920.75 & 271138.6 & 0.66 & \multirow[t]{2}{*}{1.25} & 22.15 & \multirow[t]{2}{*}{$\mathrm{P}$} \\
\hline GALEX & 155920.73 & 271140.2 & - & & 0.092125 & \\
\hline \#42, GMRT-J 155930+270530 & 155930.92 & 270530.7 & 0.76 & \multirow[t]{2}{*}{1.49} & 22.18 & \multirow[t]{2}{*}{$\mathrm{P}$} \\
\hline SDSS & 155930.98 & 270529.1 & 18.4 & & 0.088270 & \\
\hline
\end{tabular}



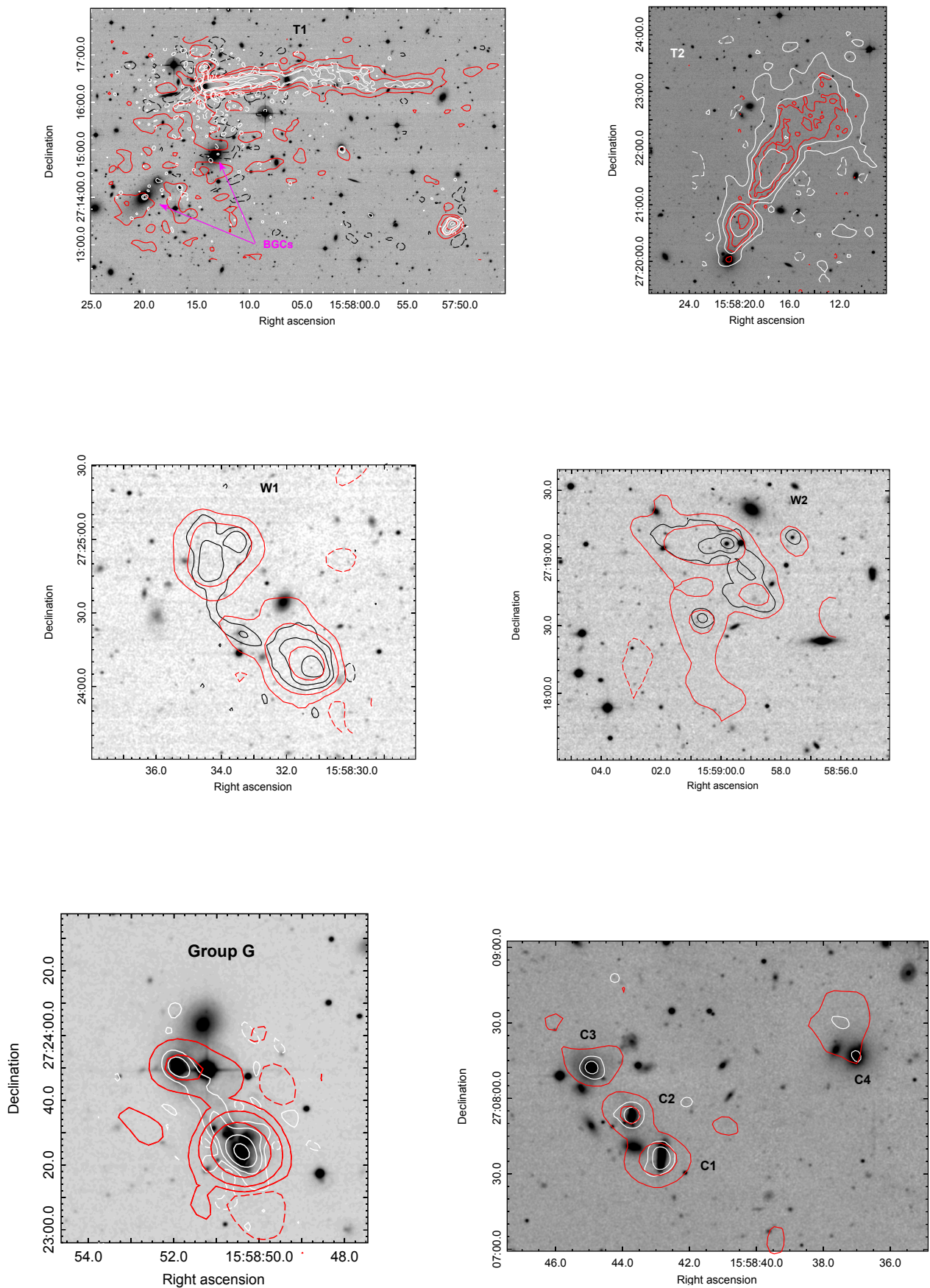

Fig. A.1. Overlays between a CFHT MegaCam g-band image and the radio emission at $608 \mathrm{MHz}\left(\right.$ resolution of 5.2" $\times 4.5^{\prime \prime}, \mathrm{PA} 52.6^{\circ}$ ) and at $234 \mathrm{MHz}$ (resolution of $12.8^{\prime \prime} \times 11.6^{\prime \prime}, \mathrm{PA} 67.3^{\circ}$ ). In all panels the $608 \mathrm{MHz}$ contours are spaced by a factor of 4 starting from $\pm 0.15 \mathrm{mJy} \mathrm{b}^{-1}$ and the $234 \mathrm{MHz}$ contours are spaced by a factor of 4 starting from $\pm 0.75 \mathrm{mJy} \mathrm{b}^{-1}$. Panels are as follows. Upper left: T1 (B2 1556+27), 608 MHz contours are indicated in white (negative dashed), $234 \mathrm{MHz}$ contours are indicated in red (negative dashed black). The two BCGs are shown. Upper right: T2 (B2 1556+27), $608 \mathrm{MHz}$ contours are shown in red (negative dashed), $234 \mathrm{MHz}$ contours are shown in white (negative dashed). Central left: W1, $608 \mathrm{MHz}$ contours are shown in black (negative dashed), $234 \mathrm{MHz}$ contours are indicated in red (negative dashed red). Central right: W2, $608 \mathrm{MHz}$ contours are indicated in black (negative dashed), $234 \mathrm{MHz}$ contours are indicated in red (negative dashed). Bottom left: Group G, $608 \mathrm{MHz}$ contours are shown in white (negative dashed), $234 \mathrm{MHz}$ contours are shown in red (negative dashed). Bottom right: Group C, $608 \mathrm{MHz}$ contours are indicated in white (negative dashed), $234 \mathrm{MHz}$ contours are shown in red (negative dashed). 
T. Venturi et al.: The two-component giant radio halo in A 2142

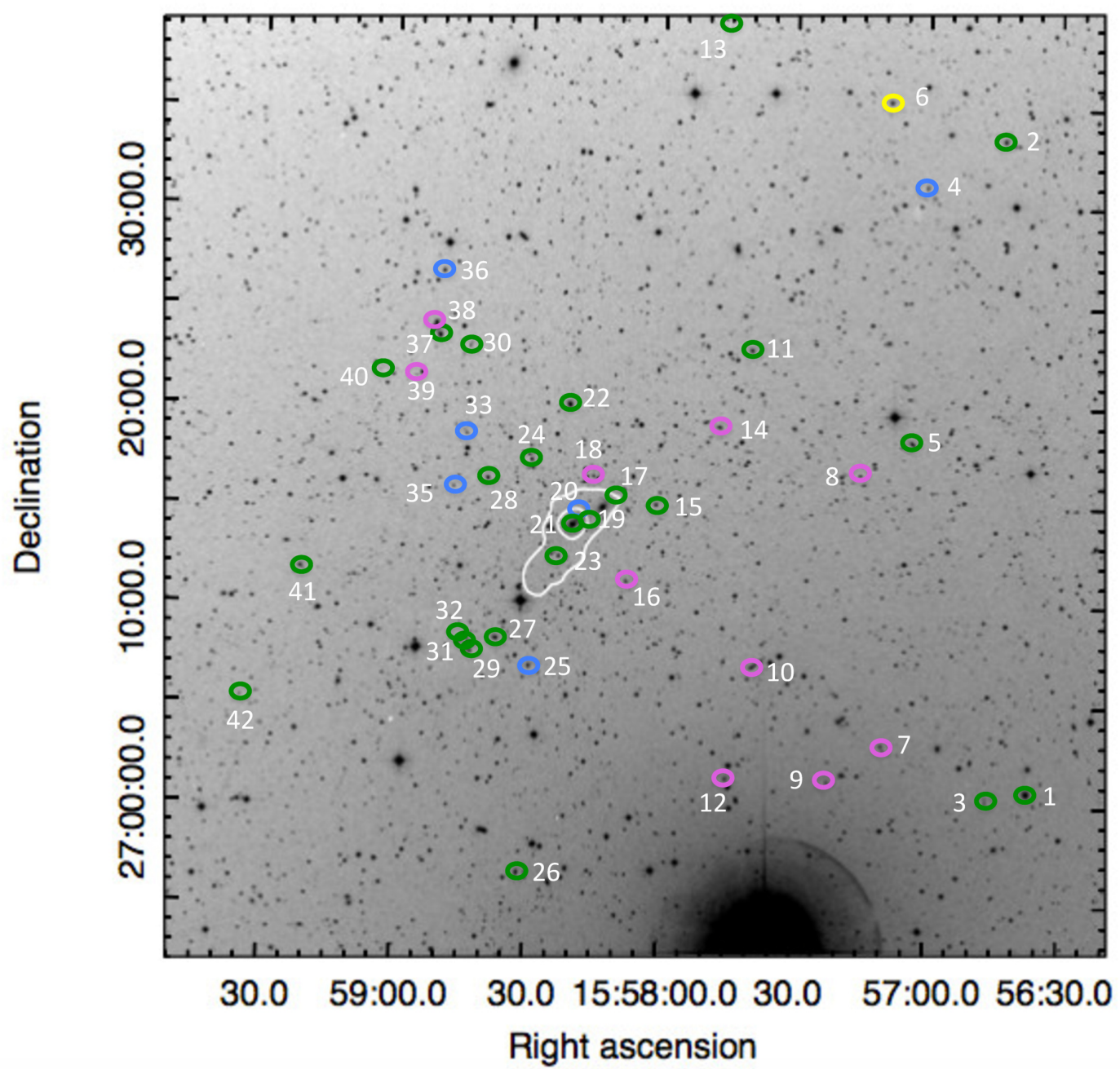

Fig. A.2. Location of the radio galaxies in A 2142 listed in Table A.1 on the DSS2 red plate (circles). The numbering follows that of the table. The $1377 \mathrm{MHz}$ radio contours of the halo are shown in white $\left(0.8\right.$ and $1.6 \mathrm{mJy} \mathrm{b}^{-1}$, resolution $\left.60^{\prime \prime} \times 60^{\prime \prime}\right)$. Different redshift intervals are colour coded as follows: blue $=0.075-0.085$; green $=0.085-0.095$; magenta $=0.095-0.105$; and yellow $=0.105-0.125$. 